\title{
$X$-ray flux in the SED modelling: An application of X-CIGALE in the XMM-XXL field
}

\author{
G. Mountrichas ${ }^{1}$, V. Buat ${ }^{2,3}$, G. Yang ${ }^{4,5}$, M. Boquien ${ }^{6}$, D. Burgarella ${ }^{2}$, L. Ciesla ${ }^{2}$ \\ ${ }^{1}$ Instituto de Fisica de Cantabria (CSIC-Universidad de Cantabria), Avenida de los Castros, 39005 Santander, Spain e-mail: \\ gmountrichas@gmail.com \\ 2 Aix Marseille Univ, CNRS, CNES, LAM Marseille, France. e-mail: veronique.buat@lam. fr \\ 3 Institut Universitaire de France (IUF) \\ ${ }^{4}$ Department of Physics and Astronomy, Texas A\&M University, College Station, TX 77843-4242, USA \\ 5 George P. and Cynthia Woods Mitchell Institute for Fundamental Physics and Astronomy, Texas A\&M University, College Station, \\ TX 77843-4242, USA \\ ${ }^{6}$ Centro de Astronomía (CITEVA), Universidad de Antofagasta, Avenida Angamos 601, Antofagasta, Chile
}

November 19, 2020

\section{ABSTRACT}

X-CIGALE, built upon the spectral energy distribution (SED) code of CIGALE, implements important new features: the code accounts for obscuring material in the polars of the AGN and has the ability to fit X-ray fluxes. In this work, we use $\sim 2500$ spectroscopic, X-ray AGN from the XMM-XXL-North field and examine the improvements the new features bring in the SED modelling analysis. Based on our results, X-CIGALE successfully connects the X-ray with the UV luminosity in the whole range spanned by our sample $\left(\log \mathrm{L}_{\mathrm{X}}(2-10 \mathrm{keV})=(42-46) \mathrm{erg} \mathrm{s}^{-1}\right)$. The addition of the new features globally improves the efficiency of X-CIGALE in the estimation and characterization of the AGN component. The classification into type 1 and type 2 based on their inclination angle is improved, especially at redshifts lower than 1 . The statistical significance of the AGN fraction, frac ${ }_{\mathrm{AGN}}$, measurements is increased, in particular for luminous X-ray sources $\left(\mathrm{L}_{\mathrm{X}}>10^{45} \mathrm{erg} \mathrm{s}^{-1}\right)$. These conclusions hold under the condition that (mid-) IR photometry is available in the SED fitting process. The addition of polar dust increases the AGN fraction and the efficiency of the SED decomposition to detect AGN among X-ray selected sources. X-CIGALE estimates a strong AGN ( $f \operatorname{cac}_{\mathrm{AGN}}>0.3$ ) in more than $90 \%$ of the infrared selected AGN and $75 \%$ of X-ray detected AGN not selected by IR colour criteria. The latter drops to 50\% when polar dust is not included. The ability of X-CIGALE to include X-ray information in the SED fitting process can be instrumental in the optimal exploitation of the wealth of data that current (eROSITA) and future (ATHENA) missions will provide us.

\section{Introduction}

One of the major challenges in current astrophysical research is to understand the physical processes that operate on top of the dark matter distribution to produce luminous structures, such as stars and galaxies. These baryonic physics has a high level of complexity mainly because it includes electromagnetic interactions, for instance the interplay between cooling and heating of baryons (e.g. gas of dust particles) via radiative processes, or the impact of magnetic fields on charged particles. However, such processes operate on spatial scales many orders of magnitude smaller than what can be achieved by state-of-the-art cosmological simulations. Thus, assumptions have to be made on the amplitude and impact of the multitude of possible mechanisms that may affect the formation and evolution of galaxies, e.g. gas supply, gas cooling and heating, impact of stellar winds on interstellar medium. These physical processes that govern the birth and fate of galaxies are of extreme complexity. However, they are of great interest since galaxies play a pivotal role in the structure of the Universe and are unique tracers of its evolution.

One manifestation of baryonic physics is the formation of supermassive black holes (SMBHs) at the centre of galaxies. Tight correlations have been found between the mass of the SMBH and the properties of its bulge (e.g. Magorrian et al. 1998; Ferrarese \& Merritt 2000). When material is accreted onto these SMBHs it triggers them and the galaxy is called Active Galactic Nuclei (AGN). The energy released during the accretion process is also an important source of heating for both the interstellar (e.g. Mor- ganti 2017) and intergalactic medium (e.g. Kaastra et al. 2014). As a result, it has been hypothesised that SMBHs and their activity plays an important role in galaxy evolution (e.g. Brandt \& Alexander 2015).

AGN emission can be observed at different wavelengths from X-rays to radio, as different physical mechanisms produce radiation at different wavelengths. In particular, X-ray emission is a trademark of AGN activity. This emission originates from photons produced by the accretion disk that are scattered by the hot corona and emit X-rays through inverse Compton scattering. This process, dominates the X-ray emission of the host galaxy and reflects the activity of the central SMBH. Thus, X-rays are often used as a proxy of AGN power (e.g. Lusso et al. 2012, Yang et al. 2019). Important ongoing (e.g., eROSITA) and future (ATHENA) X-ray missions, will use the unique window that X-rays offer and provide us a wealth of data to study the tight connection of AGN with their host galaxies.

The multi-wavelength emission of galaxies can be studied by constructing and modelling their full spectral energy distribution (SED). This method allows to measure fundamental properties of galaxies, such as their stellar mass $\mathbf{M}_{*}$, star formation rate (SFR), dust mass and attenuation while at the same time breaks degeneracies that plague observations in narrow(er) wavelength ranges. Towards this end, a number of algorithms have been developed to perform this task that follow different approaches. A popular approach is based on the energy balance principle, i.e., the energy emitted in the infrared (IR; i.e., $5-1000 \mu \mathrm{m}$ ) 
is equal to the energy absorbed in the UV/optical wavelengths, e.g. CIGALE (Code Investigating GALaxy Emission; Burgarella et al. 2005; Noll et al. 2009, Boquien et al. 2019), ProSpect (Robotham et al. 2020), MAGPHYS (da Cunha et al. 2008), Prospector (Leja et al.2017), BAGPIPES (Carnall et al. 2018).

As mentioned, AGN play an important role in galaxy evolution and their presence affects many parts of the electromagnetic spectrum. Thus, a number of the aforementioned SED fitting algorithms, have added an AGN component in the fitting process (e.g. CIGALE, ProSpect) to separate AGN and galaxy emission. SED algorithms that don't include an AGN SED component and only account for low luminosity/obscured AGN are biased against luminous/unobscured sources and underestimate the contribution of the AGN IR emission to the total IR galaxy emission.

In a recent paper, Yang et al. (2020) presented a new branch of the CIGALE code, named X-CIGALE. Compared to CIGALE, X-CIGALE is supplemented with the modelling of AGN X-ray emission and the inclusion of polar dust. Polar dust accounts for extinction of ultraviolet (UV) and optical radiation, that is commonly found, in particular, in X-ray selected AGN (Bongiorno et al. 2012). SED fitting algorithms that include Xray information could be instrumental in the exploitation and interpretation of the large datasets that X-ray surveys will provide.

The goal of this paper is to use the new capabilities of the X-CIGALE code on one of the largest X-ray samples available (XMM-XXL; Pierre et al.|2016). XXL offers a significantly wider luminosity baseline that extents to higher luminosities compared to the fields studied in Yang et al.(2020). Additionally, the size of the database allows to draw statistically robust conclusions in our tests. The physical properties of the XXL AGN and their host galaxies, using the CIGALE code, have been the topic of previous studies (e.g. Masoura et al.2018, 2020, Masoura et al. submitted). In this work, we shall focus on the effect of the new features of X-CIGALE on important SED fitting parameters. Our main purpose, is to examine how reliably the algorithm connects the X-ray flux with the UV luminosity and the rest of the other wavelengths, how accurately X-CIGALE can reproduce the X-ray properties of the AGN and what improvements the new additions bring in its efficiency on the SED decomposition.

\section{Data}

In this section, we describe the X-ray AGN sample used in our analysis and the methodology we follow to obtain optical and IR identifications.

\subsection{The X-ray AGN sample}

Throughout our work, we use spectroscopic X-ray AGN from the XMM-XXL field (Pierre et al.2016). XXL is an international project based around an XMM Very Large Programme surveying two $25 \mathrm{deg}^{2}$ extragalactic fields. It has a depth of $\sim 6 \times 10^{-15}$ $\operatorname{erg~cm} \mathrm{cm}^{-2} \mathrm{~s}^{-1}$ in the [0.5-2] keV band for point-like sources, with an exposure time of about $10 \mathrm{ks}$ per XMM pointing. $8445 \mathrm{X}$-ray sources have been detected in the equatorial subregions (XMMXXL-N; Liu et al. 2016). 5294 of them have SDSS counterparts. Spectroscopic redshifts are available for 2512 AGN (Menzel et al.2016) from SDSS-III/BOSS (Eisenstein et al. 2011; Smee et al. 2013; Dawson et al. 2013). These spectroscopic sources are used in our analysis. Their redshift distribution is presented in Fig. 1 .

\subsection{IR Photometry}

In addition to the optical (SDSS) photometry available for all our sources, we also search for counterparts in the near-IR, midIR and far-IR part of the spectrum. Mid-IR (allWISE; Wright et al. 2010) and near-IR photometry from the Visible and Infrared Survey Telescope for Astronomy (Emerson et al. 2006. VISTA) have been obtained using the Likelihood Ratio method (e.g. Sutherland \& Saunders 1992) as implemented in Georgakakis et al. (2011). The process is described in detail in Georgakakis et al. (2017).

We also use catalogues produced by the HELP ${ }^{1}$ collaboration to complement our mid-IR photometry with Spitzer (Werner et al. 2004) observations and add far-IR counterparts. HELP provides homogeneous and calibrated multiwavelength data over the Herschel Multitiered Extragalactic Survey (HerMES, Oliver et al. 2012) and the H-ATLAS survey (Eales et al. 2010). The strategy adopted by HELP is to build a master list catalog of objects as complete as possible for each field (Shirley et al. 2019) and to use the near-IR sources from IRAC surveys as prior information for the IR maps. The XID+ tool (Hurley et al. 2017), developed for this purpose, uses a Bayesian probabilistic framework and works with prior positions. At the end, a flux is measured, in a probabilistic sense, for all the near-IR sources of the master list. The XMM-LSS field was covered by two Spitzer surveys, SpUDS (Spitzer UKIDSS Ultra Deep Survey, Caputi et al. 2011) and SWIRE/SERVS (Lonsdale et al. 2003). The prior positions are defined with the SpUDS and SWIRE/SERVS surveys and the fluxes are measured for the Spitzer MIPS/24 microns, and Herschel PACS and SPIRE bands. In this work, only the MIPS and SPIRE fluxes are considered, given the much lower sensitivity of the PACS observations for this field (Oliver et al. 2012).

The W1, W2 photometric bands of WISE nearly overlap with IRAC1, IRAC2 from Spitzer. When a source has been detected by both IR surveys, we only consider the photometry with the highest signal-to-noise ratio (SNR). Similarly, when both W4 and MIPS photometry is available, we consider only the latter due to the higher sensitivity of Spitzer compared to WISE. The number of sources available in each photometric band of our final sample is presented in Table 1 . Table 2, shows the sensitivity of each photometric band, used in our analysis.

\section{Analysis}

$\mathrm{X}$-CIGALE requires the intrinsic, i.e. unabsorbed, flux of the $\mathrm{X}$ ray AGN. In this section, we describe how we estimate the $\mathrm{X}$ ray absorption of the sources to infer their intrinsic X-ray flux. We also describe the modules and parameters used for the SED fitting process and present our final sample.

\subsection{Estimation of the $X$-ray properties}

To estimate the intrinsic, X-ray flux of each source, we need to measure their hydrogen column density, $\mathrm{N}_{\mathrm{H}}$, that quantifies the X-ray absorption. Towards this end, we use the number of photons in the soft $(0.5-2.0 \mathrm{keV})$ and the hard $(2.0-8.0 \mathrm{keV})$ bands that are provided in the Liu et al. (2016) catalogue. We

\footnotetext{
1 The Herschel Extragalactic Legacy Project (HELP, http://herschel.sussex.ac.uk/) is a European funded project to analyse all the cosmological fields observed with the Herschel satellite. All the HELP data products can be accessed on HeDaM (http://hedam.lam.fr/HELP/)
} 
Table 1: Number of available spectroscopic sources in different photometric bands. 2342/2509 ( 93\%) of the sources, have optical and mid-IR photometry available (either W1,W2 or IRAC1, IRAC2). 1276 ( 51\%) have far-IR photometry available (and mid-IR).

\begin{tabular}{cccccccc}
\hline Total & W1, W2 / IRAC1, IRAC2 & IRAC3 & IRAC4 & W3 & MIPS1 & Herschel & all bands \\
\hline 2509 & 2342 & 1122 & 1135 & 1240 & 1298 & 1276 & 408 \\
\hline
\end{tabular}

Table 2: $5 \sigma$ sensitivity (mag) of the photometric bands, used in our analysis.

\begin{tabular}{cccccc}
\hline u, g, r, i, z & J, H, K & W1, W2, W3, W4 & IRAC1, IRAC2, IRAC3, IRAC4 & MIPS1 & SPIRE \\
\hline $22.15,23.13,22.70,22.20,20.71$ & $20.6,19.8,18.5$ & $16.83,15.60,11.32,8.0$ & $24.09,23.66,21.90,21.85$ & 19.8 & $13.9,13.5,13.2$ \\
\hline
\end{tabular}

then apply a Bayesian approach to estimate the hardness ratio, $\mathrm{HR}=\frac{\mathrm{H}-\mathrm{S}}{\mathrm{H}+\mathrm{S}}$, of each source, where $\mathrm{H}$ and $\mathrm{S}$ are the counts in the soft and hard bands, respectively. Specifically, we utilise the Bayesian Estimation of Hardness Ratios code (BEHR; Park et al.2006), that accounts for the Poissonian nature of the observations. These HR values are then inserted in the PIMMS tool (Portable, Interactive, Multi-Mission Simulator; Mukai 1993) to estimate the $\mathrm{N}_{\mathrm{H}}$ of each source. In this process, we assume that the power law of the X-ray spectra have a fixed photon index, $\Gamma=1.8$ and the value of the galactic $\mathrm{N}_{\mathrm{H}}$ is $\mathrm{N}_{\mathrm{H}}=10^{20.25} \mathrm{~cm}^{-2}$. The distribution of $\mathrm{N}_{\mathrm{H}}$ of our AGN sample is presented in Fig. 2. Due to the Bayesian nature of our calculations, some $\log \mathrm{N}_{\mathrm{H}}$ values are below 20.25 , i.e. the galactic absorption.

The X-ray absorption that was estimated through PIMMS and based on which the intrinsic X-ray flux is inferred, does not necessarily correlate with the dust that the observed mid- and farIR emission exhibits. Although there are studies that have found a correlation between optical/IR obscuration and X-ray absorption (e.g. Civano et al. 2012), these correlation presents a large scatter (e.g. Jaffarian \& Gaskell 2020). This scatter is attributed to e.g. i) the X-ray column density variability (e.g. Reichert et al. 1985: Yang et al. 2016), ii) absorbing material located at galactic scales (e.g. Malizia et al. 2020) that is mainly heated by star formation rather than $A G N$, iii) the fact that different obscuration criteria are sensitive to different amounts of obscuration (e.g. Masoura et al. 2020) and iv) a dust-to-gas ratio that is different from the Galactic. Therefore, a source may present X-ray absorption without being optical red (dust-free gas), may also be heavily X-ray absorbed with broad UV/optical lines (e.g. Li et al. 2019), can be optically red, with absorbed AGN emission in its SED without being X-ray absorbed (e.g. Masoura et al. 2020) and can present higher X-ray absorption than what is expected from its optical extinction. Thus, we do not necessarily expect consistency between the X-ray absorption estimated through PIMMS with that from dust, estimated by X-CIGALE, by modelling the mid and far-IR emission.

\subsection{SED fitting with $X-C I G A L E$}

The fitting capabilities of CIGALE have been recently extended to X-rays with the development of X-CIGALE (Yang et al. 2020), in order to improve the characterisation of the AGN component. The X-ray emission is connected to the AGN emission at other wavelengths via the $\alpha_{\text {ox }}-L_{2500 \AA}$ relation of Just et al. (2007), where $L_{2500 \AA}$ is the intrinsic (de-reddened) UV luminosity and $\alpha_{\text {ox }}$ the spectral slope between $\mathrm{UV}(2500 \AA)$ and X-ray $(2 \mathrm{keV}), \alpha_{o x}=-0.3838 \log \left(\mathrm{L}_{2500 \AA} / \mathrm{L}_{2 \mathrm{keV}}\right)$. The contribution of $\mathrm{X}$-ray binaries is also considered and modelled as a function of SFR and stellar mass of the host galaxy. The clumpy twophase torus model, SKIRTOR, based on 3D radiation-transfer

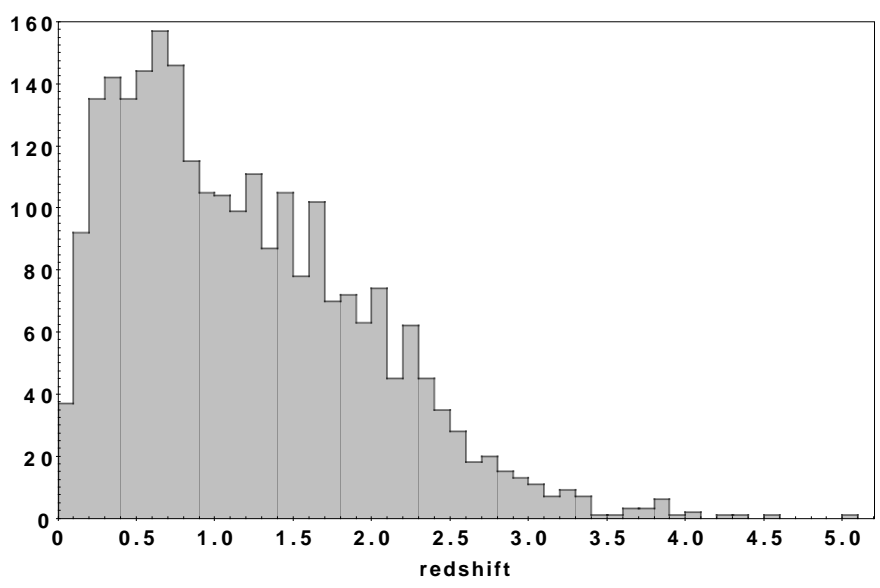

Fig. 1: The redshift distribution of the X-ray AGN sample.

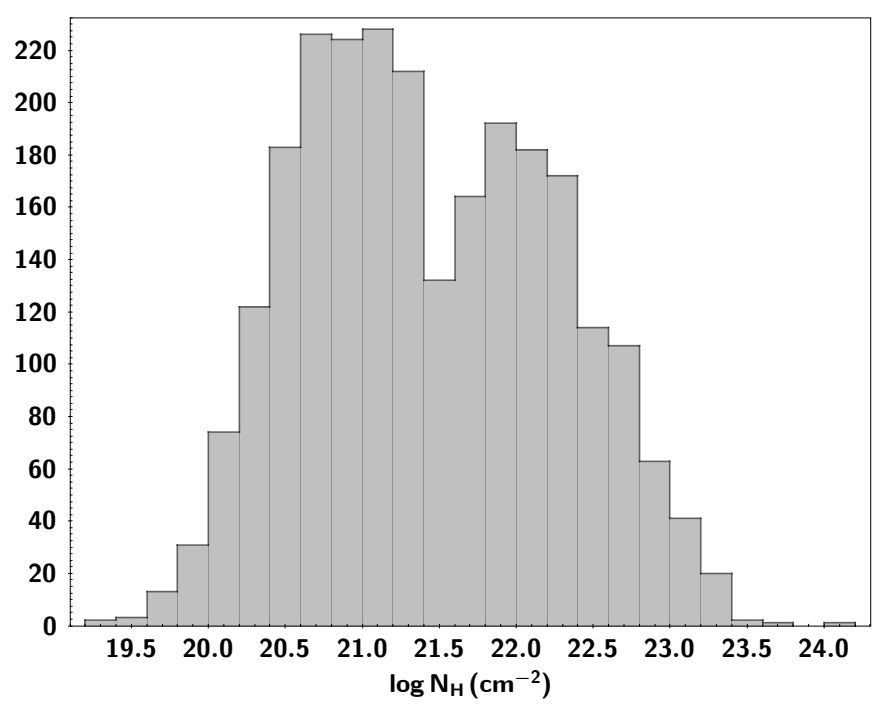

Fig. 2: Distribution of the X-ray absorption, $\mathrm{N}_{\mathrm{H}}$, of the X-ray AGN sample.

(Stalevski et al. 2012, 2016) is used for the UV to far-IR emission of the AGN with some modifications keeping the energy balance: the original emission of the accretion disc is updated with the spectral energy distribution of Feltre et al. (2012) and dust extinction and emission in the poles of type $1 \mathrm{AGN}$ is also considered (e.g. Bongiorno et al. 2012; Tristram et al. 2014; Asmus et al. 2014; Asmus 2019). We refer to Yang et al. (2020) for a full description of X-CIGALE. Here, we describe the main steps to build the models and fit our X-ray to far-IR data. The 
Table 3: The models and the values for their free parameters used by X-CIGALE for the SED fitting of our galaxy sample. For the definition of the various parameter see section 3.2

\begin{tabular}{|c|c|}
\hline Parameter & Model/values \\
\hline \multicolumn{2}{|c|}{ Star formation history: delayed model } \\
\hline e-folding time & $100,500,1000,5000$ \\
\hline Stellar age age & $500,1000,3000,5000,7000$ \\
\hline \multicolumn{2}{|c|}{ Simple Stellar population: Bruzual \& Charlot (2003) } \\
\hline Initial Mass Function & Chabrier (2003) \\
\hline Metallicity & 0.02 (Solar) \\
\hline \multicolumn{2}{|c|}{ Galactic dust extinction } \\
\hline Dust attenuation law & Calzetti et al. (2000) \\
\hline Reddening $E(B-V)$ & $0.0,0.1,0.2,0.3,0.4,0.5,0.6,0.7,0.8,0.9$ \\
\hline \multicolumn{2}{|c|}{ Galactic dust emission: Dale et al. (2014) } \\
\hline$\alpha$ slope in $d M_{d u s t} \propto U^{-\alpha} d U$ & $1.0,1.5,2.0,2.5,3.0$ \\
\hline \multicolumn{2}{|c|}{ AGN module: SKIRTOR) } \\
\hline Torus optical depth at 9.7 microns $\tau_{9.7}$ & 7.0 \\
\hline Torus density radial parameter $\mathrm{p}\left(\rho \propto r^{-p} e^{-q|\cos (\theta)|}\right)$ & 1.0 \\
\hline Torus density angular parameter $\mathrm{q}\left(\rho \propto r^{-p} e^{-q|\cos (\theta)|}\right)$ & 1.0 \\
\hline Angle between the equatorial plan and edge of the torus & $40^{\circ}$ \\
\hline Ratio of the maximum to minimum radii of the torus & 20 \\
\hline Viewing angle & $30^{\circ}$ (type 1$), 70^{\circ}$ (type 2 ) \\
\hline AGN fraction & $0.0,0.01,0.1,0.2,0.3,0.4,0.5,0.6,0.7,0.8,0.9,0.99$ \\
\hline Extinction law of polar dust & SMC \\
\hline$E(B-V)$ of polar dust & $0.0,0.01,0.02,0.03,0.05,0.1,0.2,0.4,0.6,1.0,1.8$ \\
\hline Temperature of polar dust (K) & 100 \\
\hline Emissivity of polar dust & 1.6 \\
\hline \multicolumn{2}{|c|}{ X-ray module } \\
\hline AGN photon index $\Gamma$ & 1.8 \\
\hline Maximum deviation from the $\alpha_{o x}-L_{2500 \AA}$ relation & 0.2 \\
\hline LMXB photon index & 1.56 \\
\hline HMXB photon index & 2.0 \\
\hline
\end{tabular}

modules and input parameters we use in our analysis are presented in Table 3

\subsubsection{Galaxy emission}

For the sake of simplicity and since we will not study in detail the SFR properties of our sources, we adopt a simple star formation history (SFH). The galaxy component is built using a delayed SFH $($ SFR $\propto \mathrm{t} \times \exp (-\mathrm{t} / \tau))$. We checked that the addition of a recent burst (e.g. Masoura et al. 2018; Małek et al. 2018) did not change our results. The stellar emission is modelled using the Bruzual \& Charlot (2003) template. A Chabrier (2003) initial mass function (IMF) is used with metallicity equal to 0.02. The stellar emission is attenuated with the Calzetti et al. (2000) attenuation law. The IR SED of the dust heated by stars is implemented with the Dale et al. (2014) template.

\subsubsection{AGN emission}

The AGN emission is modelled using the SKIRTOR template (Stalevski et al. 2012, 2016). A polar dust component is added that is modelled as a dust screen absorption and a grey-body emission. As in Yang et al. (2020), we adopt the SMC extinction curve (Small Magellanic Cloud; Prevot et al. 1984) with $\mathrm{E}_{(\mathrm{B}-\mathrm{V})}$ as a free input parameter (see section 5 for the effects of $E_{(B-V)}$ on SED fitting). The grey-body dust re-emission is parametrised with a temperature of $100 \mathrm{~K}$ and an emissivity index of 1.6. This emission is supposed to be isotropic and thus contributes to the
IR emission of both type 1 and type 2 AGNs. We will discuss in section 4.3 the effect of a modification of the dust temperature and of the extinction curve. The contribution of the AGN to the total SED is quantified by the AGN fraction, frac $\mathrm{AGN}_{\mathrm{AGN}}$, defined as the fraction of the total IR emission coming from the AGN. Following Yang et al. (2020) two viewing angles are considered, $30^{\circ}$ and $70^{\circ}$, for type 1 and type 2 AGN, respectively.

The photon index $\Gamma$ of the AGN X-ray spectrum is fixed to 1.8 , for consistency with the value used for the estimation of $\mathrm{N}_{\mathrm{H}}$ (see section 3.1). We adopt a maximal acceptable value $\left|\Delta \alpha_{\text {ox }}\right|_{\max }=0.2$ for the dispersion of the $\alpha_{\text {ox }}-L_{2500 \AA}$ relation (Risaliti \& Lusso 2017). This value is also adopted in Yang et al. (2020) and corresponds to $\approx 2 \sigma$ scatter in the $\alpha_{o x}-\mathrm{L}_{2500 \AA}$ relation (Just et al. 2007). Nine values of $\alpha_{\mathrm{ox}}$ are defined (from -1.9 to -1.1 with a step of 0.1 ) and X-CIGALE adds an X-ray flux to each (UV to far-IR AGN SED, $\alpha_{\text {ox }}$ ) pair, multiplying the number of AGN models by 9 .

\subsubsection{SED fitting and parameter estimation}

The spectral models described above are normalised to the creation of $1 \mathrm{M}_{\odot}$. The first step of the fitting process is to scale each model to the observations by minimising its $\chi^{2}$ (Noll et al. 2009, Boquien et al. 2019). After this scaling operation extensive quantities as luminosities are defined, including the intrinsic $L_{2500 \AA}$ which is an output of the SKIRTOR module: models which do not satisfy the $\alpha_{\mathrm{ox}}-L_{2500 \AA}$ relation and its maximum dispersion are discarded. The likelihood of the remaining models are computed and parameter values are estimated from 
Table 4: Number of AGN that satisfy our selection criteria (section 3.3 for different configurations of the SED fitting process.

\begin{tabular}{ccccc}
\hline Total & $\mathrm{f}_{\mathrm{X}}$, polar dust & no $\mathrm{f}_{\mathrm{X}}$, polar dust & $\mathrm{f}_{\mathrm{X}}$, no polar dust & no $\mathrm{f}_{\mathrm{X}}$, no polar dust \\
\hline 2509 & 2348 & 2274 & 2337 & 2274 \\
\hline
\end{tabular}
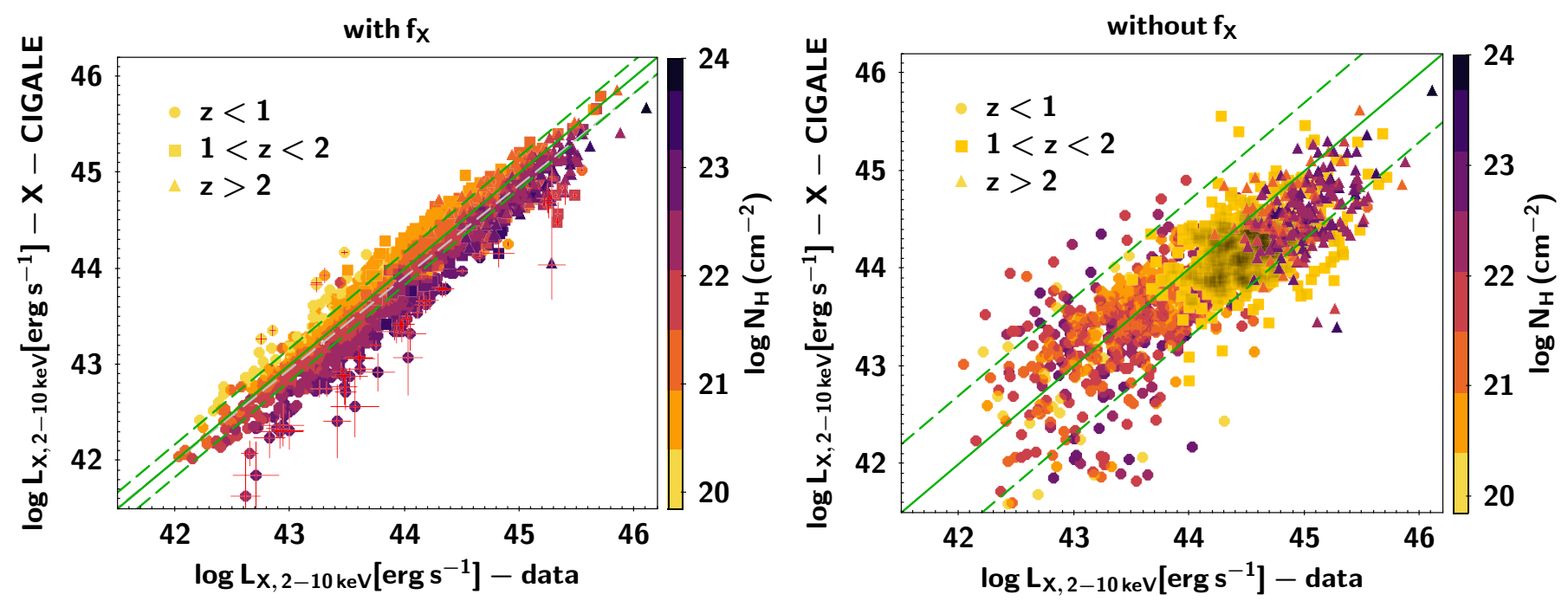

Fig. 3: Comparison of the intrinsic, X-ray luminosity in the 2-10 keV band estimated by the SED fitting with that from the input catalogue. Green, solid and dashed lines present the 1:1 correspondence and the errors added in quadrature of the X-ray luminosity calculations from X-CIGALE and those quoted in the X-ray catalogue (Menzel et al. 2016), respectively. The grey, dashed line shows the $\chi^{2}$ fit of the calculations. Symbols are colour coded, based on their NH values. Left panel: Results with the X-ray flux included in the SED. X-CIGALE calculations are consistent with those from the input catalogue, at all luminosities spanned by our sample. There are 70 sources that their $\mathrm{L}_{\mathrm{X}}$ calculation differs by more than $0.5 \mathrm{dex}$ from their input values (shown with errorbars, see text for more details). Right panel: The X-ray flux is not included in the SED. In this case, the scatter is significantly larger while the error of the $\mathrm{L}_{X}$ calculations from $\mathrm{X}-\mathrm{CIGALE}$, increases by $\approx 4 \times$.

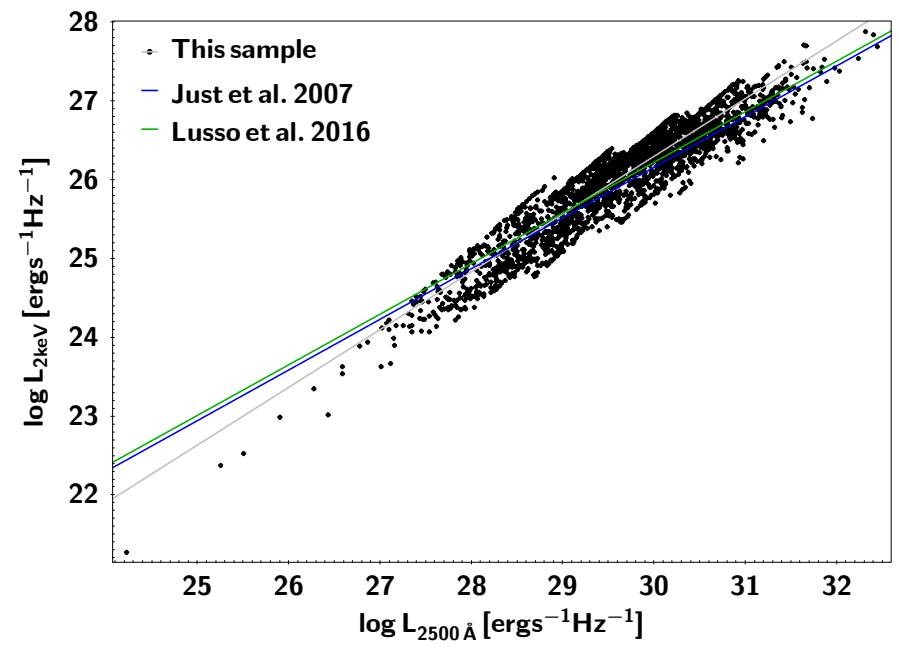

Fig. 4: $\mathrm{L}_{2 \mathrm{keV}}$ vs. $\mathrm{L}_{2500 \AA}$ relation. Blue line presents the Just et al. (2007) relation, that X-CIGALE uses to connect the X-ray flux with the UV (2500 ̊) luminosity. Green line shows the $\mathrm{L}_{2 \mathrm{keV}}$ vs. $\mathrm{L}_{2500 \AA}$ from Lusso \& Risaliti (2016). For comparison, we also plot the fit from our calculations (grey line).

their marginalised probability distribution function (likelihood weighted mean and standard deviation). The best model corresponding to each observed SED is also an output of the code.

\subsubsection{Mock catalogues}

The validity of a parameter estimation can be assessed through the analysis of a mock catalogue. When this option is chosen, the code considers the best fit of each object and a mock catalogue is built. Each best flux being modified by injecting noise taken from a Gaussian distribution with the same standard deviation as the observed flux. The mock data are then analysed in the same way as the observed data and the accuracy of the parameter estimation can be tested by comparing input (ground truth) and output (estimated) values. Our tests using the results from the mock catalogues are presented in the Appendix.

\subsection{Final sample}

In our analysis, we compare the estimations of the frac $_{\mathrm{AGN}}$, namely the value of the best model and then mean of the probability density function (PDF) distribution, to select the Xray AGN with the most reliable SED measurements. For that purpose, we apply the following criteria: we exclude sources for which their best AGN fraction values are zero while their Bayesian AGN fraction is greater than 0.4. Such a large difference between the best and the Bayesian values, is a strong indicator that the code failed to accurately fit the SED since the PDF distribution is not consistent with the best model. We also exclude AGN with reduced $\chi^{2}, \chi_{\text {red }}^{2}>10$ from the SED fitting process. These two criteria exclude $\sim 4-7 \%$ of our sources. Finally, we remove from our final sample, sources with $\mathrm{L}_{\mathrm{X}}<10^{42} \mathrm{erg} \mathrm{s}^{-1}$ to minimise contamination from inactive 


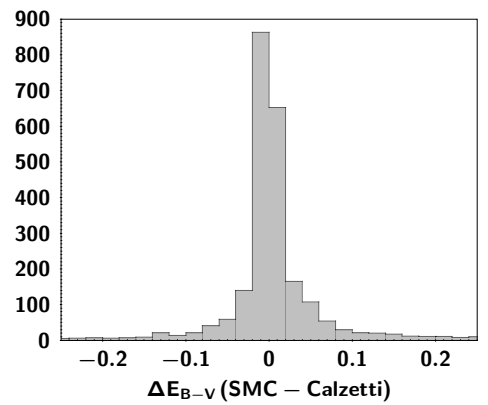

(a)

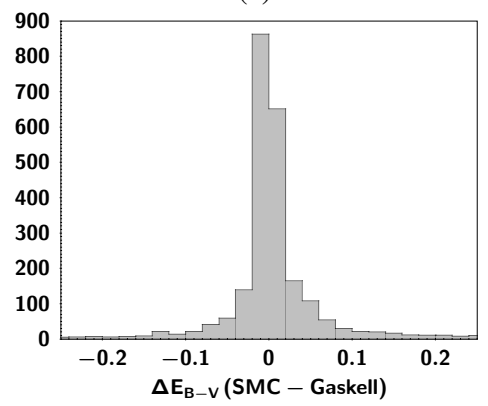

(b)

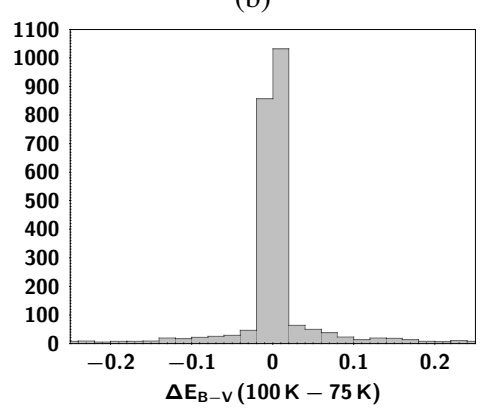

(c)

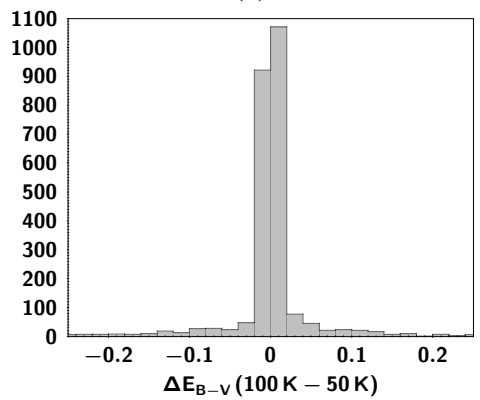

(d)

Fig. 5: Distribution of the difference of polar dust measurements, for different extinction laws (panels a, b) and grey-body dust temperatures (panels c, d). All distributions are highly peaked at zero, which indicates that polar dust calculations are not sensitive to the choice of these parameters.

galaxies. Table 4 presents the number of sources that satisfy our selection criteria for each configuration of the SED fitting process used in our analysis.

\section{X-CIGALE performance}

\subsection{X-ray luminosity}

First, we compare the intrinsic, X-ray luminosities of the AGN estimated by X-CIGALE with those from the input catalogue.

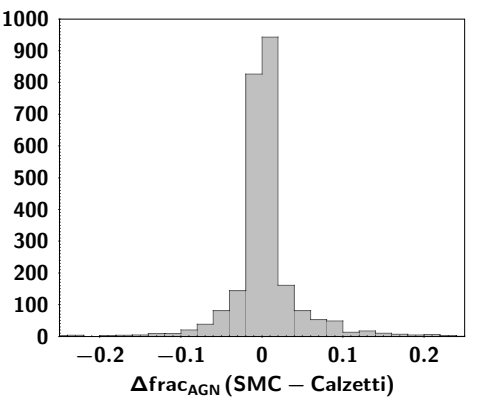

(a)

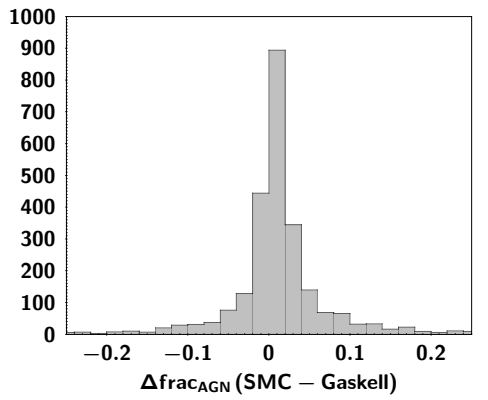

(b)

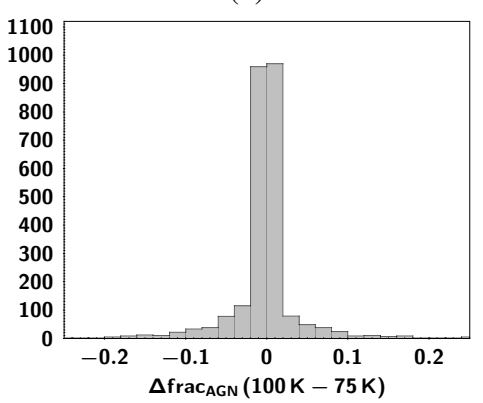

(c)

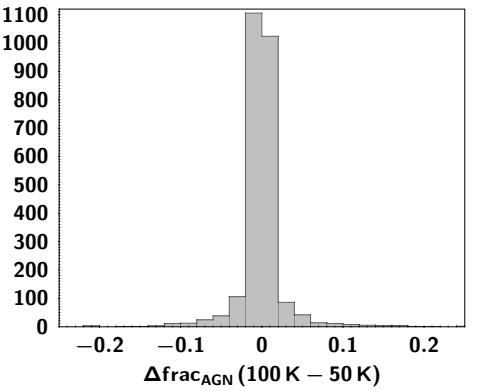

(d)

Fig. 6: Same as in the previous plot, but for the AGN fraction. Different extinction laws (panels a, b) and grey-body dust temperatures (panels $\mathrm{c}, \mathrm{d}$ ) do not affect the frac $\mathrm{AGN}_{\mathrm{AN}}$ measurements.

The results are presented in Fig. 3 X-CIGALE estimates are consistent with those from the input catalogue, at all luminosities spanned by our sample. A $\chi^{2}$ fit (grey, dashed line) gives $\log \mathrm{L}_{\mathrm{X}, \mathrm{X} \text {-CIGALE }}=(0.962 \pm 0.023) \log \mathrm{L}_{\mathrm{X}, \mathrm{data}}+1.694 \pm 0.085$. Green, dashed lines present the errors added in quadrature of the X-ray luminosity calculations from X-CIGALE and those quoted in the X-ray catalogue (Menzel et al. 2016). There are 70 outliers, i.e., sources for which their $\mathrm{L}_{\mathrm{X}}$ calculations differ by more than $0.5 \mathrm{dex}$ from their input values (shown with errorbars). The vast majority of sources that X-CIGALE underestimates their $\mathrm{L}_{X}$ value by more than $0.5 \mathrm{dex}$ are AGN with 
high X-ray absorption $\left(\mathrm{N}_{\mathrm{H}}>10^{22.5-23.0} \mathrm{~cm}^{-2}\right) .82 \%$ of these sources also lack far-IR photometry and $57 \%$ do not have IR coverage above $8 \mu \mathrm{m}$. Thus, a possible explanation for the discrepant $\mathrm{L}_{\mathrm{X}}$ values could be that our $\mathrm{N}_{\mathrm{H}}$ estimations are not accurate and/or the lack of available IR photometry does not allow $\mathrm{X}$-CIGALE to properly fit these SEDs. Lowering the $\mathrm{L}_{\mathrm{X}}$ difference threshold to 0.3 dex to characterise a source as outlier, leads to the same conclusions. On the other hand, those sources that XCIGALE overestimates their value by more than $0.5 \mathrm{dex}(<0.4 \%$ of the total sample), do not present any signs of $\mathrm{X}$-ray absorption $\left(\mathrm{N}_{\mathrm{H}}<10^{21.5} \mathrm{~cm}^{-2}\right)$. The quality of the SED fitting of these systems is good, based on the $\chi_{\text {red }}^{2}$ values $\left(\chi_{\text {red }}^{2} \lesssim 2\right)$. SED analysis reveals that the AGN emission is obscured in the optical wavelengths. The optical/mid-IR criteria of Yan et al. (2013), do not classify these AGN as optically red sources. Furthermore, their optical spectra present broad lines. Thus, there are no indications to corroborate with X-CIGALE that these AGN are absorbed. Therefore, we do not find a plausible explanation for these outliers. However, they are only $<0.4 \%$ of our sample $(\sim 1 \%$ if we lower the threshold of the $\mathrm{L}_{\mathrm{X}}$ difference to $0.3 \mathrm{dex}$ ).

On the right panel of Fig. 3, we plot the X-ray luminosity calculations of X-CIGALE when the X-ray flux is not included in the SED vs. the $\mathrm{L}_{X}$ from the input catalogue. In this case, the scatter is significantly larger compared to the left panel while the average error of the $\mathrm{L}_{X}$ estimates from X-CIGALE, increases by $\approx 4 \times$ (green lines). When there is no $\mathrm{f}_{X}$ in the SED, the $\mathrm{L}_{\mathrm{X}}$ estimates should not be taken at face value. X-CIGALE uses the AGN module (SKIRTOR) to output plausible $\mathrm{L}_{2500 \AA}$ values. Since there is no X-ray information to further constrain the $\mathrm{L}_{2500 \AA}$ parameter by connecting it to the observed X-ray flux, the algorithm provides $\mathrm{L}_{\mathrm{X}}$ estimations, using the full range of $\alpha_{\text {ox }}$ (allowed by the Just et al. relation and the $\alpha_{\text {ox }}$ dispersion) and weighs over these possible values. Thus, although the code provides $\mathrm{L}_{\mathrm{X}}$ estimates, these values should be taken with caution.

\subsection{The efficiency of X-CIGALE to connect the X-ray - UV luminosity}

As mentioned, $\alpha_{\text {ox }}, \mathrm{L}_{2500 \AA}$ and $\mathrm{L}_{2 \mathrm{keV}}$ are the three parameters that are important for X-CIGALE to connect the X-rays with the UV and thus the other wavelengths during the SED fitting process. In section A.1. we assess the efficiency of X-CIGALE to constrain these three parameters, by using the mock analysis (see section 3.2.4. In this section, we compare the algorithm's calculations of $\mathrm{L}_{2} \mathrm{keV}$ and $\mathrm{L}_{2500 \AA}$.

Fig. 4. compares the $\mathrm{L}_{2 \mathrm{keV}}$ and $\mathrm{L}_{2500 \AA}$ luminosities with the observed relations of Just et al. (2007) (blue line), used by the code as input (see section 3.2) and Lusso \& Risaliti (2016) (green line). Grey line presents the fit on our measurements. There is an overdensity of sources above the Just et al. relation (at high luminosities). This is due to selection bias. XMM-XXL has a low exposure time and therefore our X-ray sample is biased towards high luminosity sources.

\subsection{The effect of the extinction law and temperature of polar dust}

In section 3.2.2 we mentioned that for the polar dust estimation an SMC extinction curve is adopted and the grey-body dust temperature is set to $100 \mathrm{~K}$. The effect of the addition of polar dust in the SED fitting will be discussed in Section 5.4. In this section, we examine whether the adoption of different extinction curves and dust temperatures affect the polar dust contribution (through $\mathrm{E}_{\mathrm{B}-\mathrm{V}}$ ) and the AGN fraction calculations.

Apart from the SMC extinction curve, X-CIGALE includes the choice of the empirical extinction curves of Calzetti et al. (2000) and Gaskell et al. (2004). Figures 5a and 5b present the difference of the polar dust estimations between the SMC and the Calzetti et al. (2000) and the SMC and Gaskell et al. (2004) curves, respectively. Both distributions are highly peaked and therefore the choice of the extinction curve does not affect the polar dust measurements. In Figures $6 a$ and $6 \mathrm{~b}$, we repeat the same test for the AGN fraction values. Again, the adoption of different extinction curves does not affect the frac AGN $_{\text {. }}$.

In Figures 5c, 5d and Figures 6c, 6d, we test whether different temperatures for the grey-body dust re-emission affect the polar dust and AGN fraction calculations, respectively. Specifically, we plot the difference of the aforementioned parameters using values of $100 \mathrm{~K}$ (the value used throughout our analysis) as well as $75 \mathrm{~K}$ and $50 \mathrm{~K}$. All distributions are highly peaked at zero. We obtain similar results when we increase the temperature to $200 \mathrm{~K}$. We conclude that the choice of the parameters defining the grey-body re-emission does not affect our results.

\subsection{The effect of Herschel photometry}

Far-IR data combined with mid-IR photometric bands are known to improve the star-formation rate (SFR) estimations of galaxies hosting an AGN (Hatziminaoglou et al. 2009; Stanley et al. 2018. Masoura et al. 2018) since they constrain more efficiently the AGN contribution to the IR luminosity of the host galaxy. Masoura et al. (2018) used 608 X-ray AGN in XXL with Herschel detection and found that SFRs estimated without farIR photometry are systematically underestimated compared to SFRs with Herschel (see their Fig. 4). In this section, we examine the effect of Herschel photometry on our estimations. Specifically, we wish to test whether the absence of Herschel photometry affects the AGN fraction and polar dust estimates of X-CIGALE.

For this part of our analysis, we restrict our sample to only those AGN with reliable (SNR > 3) SPIRE photometry (in addition to the criteria mentioned in section 3.3). This will maximise any likely effect on the SED fits. This reduces our X-ray dataset to $328 \mathrm{AGN}$. We run X-CIGALE twice: one time using Herschel photometry and a second time without (both runs use the X-ray flux of the sources in the fitting process).

Figures $7 \mathrm{a}$ and $7 \mathrm{~b}$ present the difference of the polar dust and AGN fraction distributions, from the two runs, respectively. We notice that the addition of Herschel photometry slightly reduces the AGN fraction measurements. Although, the distribution of the difference of the AGN fraction estimations peaks at zero, there is a tail at negative values. Specifically, there are 73 sources $(\approx 22 \%$ of this sample) for which the addition of Herschel photometry reduces their AGN fraction value by more than 0.15 (which is equivalent to the error of the two frac $\mathrm{AGN}_{\mathrm{AN}}$ estimations added in quadrature, see next). This is also quantified by the mean frac ${ }_{\mathrm{AGN}}$ values, frac $\mathrm{AGN}_{\mathrm{AG} \text {,Herschel }}=0.36 \pm 0.08$ and frac $_{\mathrm{AGN}, \text { no Herschel }}=0.43 \pm 0.12$. We also notice that including far-IR photometry increases the statistical significance of the estimations from $3.6 \sigma$ to $4.5 \sigma$ (the significance is defined as the bayesian value over the error). The addition of Herschel photometry does not affect polar dust measurements. We also examine whether the addition of Herschel photometry affects other parameters estimated by the SED fitting and specifically the X-ray luminosity and the $\mathrm{L}_{2500 \AA}$. The distribution of the difference of the $\mathrm{L}_{\mathrm{X}}$ and $\mathrm{L}_{2500 \AA}$ parameters with and without far-IR photom- 


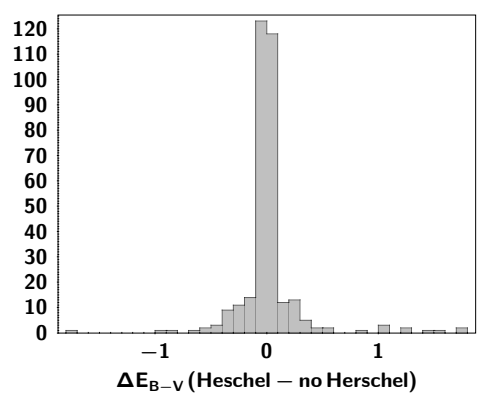

(a)

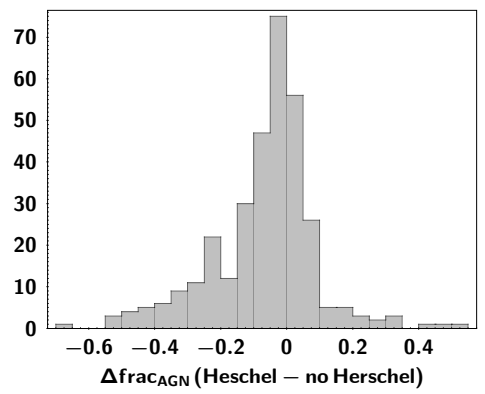

(b)

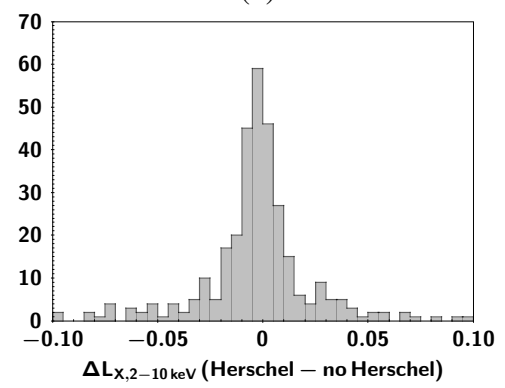

(c)

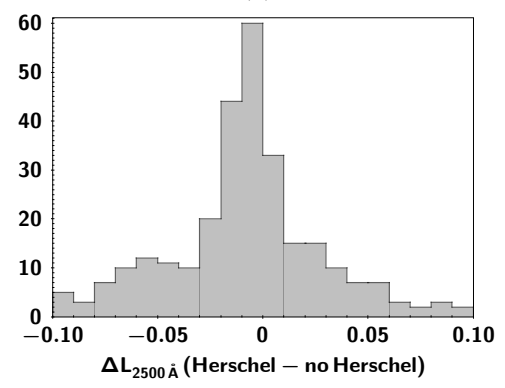

(d)

Fig. 7: Distribution of the difference of polar dust, AGN fraction and X-ray and UV luminosity measurements, with and without Herschel photometry. Polar dust and luminosity calculations are not affected by the existence of far-IR photometry. However, addition of far-IR photometry reduces the frac $\mathrm{AGN}_{\mathrm{AGN}}$. This is quantified by the mean values, frac ${ }_{\mathrm{AGN}, \text { Herschel }}=0.36 \pm 0.08$ and frac $_{\mathrm{AGN}, \text { no Herschel }}=0.43 \pm 0.12$.

etry is presented in Figures $7 \mathrm{c}$ and $7 \mathrm{~d}$. Both distributions are highly peaked at zero with small tails at both sides. Thus, inclusion of Herschel photometry does not seem to affect the estimations of these two parameters.

\section{Advantages of X-ray flux and polar dust components}

In this section, we examine the advantages that the introduction of the two new features of X-CIGALE, i.e. the X-ray flux and polar dust, bring in the SED fitting process.

\subsection{The effect of the $X$-ray flux on the AGN fraction measurements}

One of the strengths of SED decomposition is that it allows disentangling AGN emission from that of the host galaxy emission. This is a crucial issue since the reliability of any further analysis depends on how reliable the SED fitting code can perform this task. The goal of this part of our analysis is to examine if the introduction of the X-ray information in the SED, allows $\mathrm{X}$-CIGALE to improve its efficiency in estimating robust AGN fractions.

In section A.2, we assess the accuracy of X-CIGALE in the estimation of the AGN fraction. Here, we study how the addition of the the X-ray flux in the fitting process affects the AGN fraction measurements. In Fig. 8 (left panel), we plot the distribution of the AGN fraction with (black shaded area) and without (blue histogram) the X-ray flux (polar dust is included in both runs). The mean frac ${ }_{\mathrm{AGN}}$ value increases from $0.46 \pm 0.16$ to $0.49 \pm 0.14$ with the addition of $f_{X}$. To further examine the effect of the X-ray flux in the AGN fraction estimates, in the right panel we plot the difference of the AGN fraction with and without X-ray flux, for different luminosity bins. The distributions peak at zero, in all cases. However, at high X-ray luminosities $\left(\mathrm{L}_{\mathrm{X}}>10^{43} \mathrm{erg} \mathrm{s}^{-1}\right)$ a tail starts to appear at positive values, i.e., the AGN fraction tends to be higher when the X-ray flux is included in the fitting process. This tail becomes more prominent at the highest luminosity bin. Specifically, for AGN with $\mathrm{L}_{X}>10^{45} \mathrm{erg} \mathrm{s}^{-1}$ the mean AGN fraction increases from $0.58 \pm 0.16$ without $X$-ray flux to $0.65 \pm 0.13$ with $\mathrm{X}$-ray flux. The opposite trend is present for low luminosity AGN $\left(10^{42}<\mathrm{L}_{X}<10^{43} \mathrm{erg} \mathrm{s}^{-1}\right)$. In this case, the mean frac $\mathrm{AGN}_{\mathrm{AG}}$ reduces from $0.28 \pm 0.13$ to $0.23 \pm 0.09$ with the X-ray flux.

The statistical significance of the AGN fraction measurements improves with the inclusion of the X-ray flux, as can be seen by the numbers presented above. This is also illustrated in Fig. 9, where we plot the difference of the significance, $\Delta \sigma$, of the AGN fraction estimations with and without X-ray information, for sources with $\mathrm{L}_{\mathrm{X}}>10^{45} \mathrm{erg} \mathrm{s}^{-1}$ and difference in frac $_{\mathrm{AGN}}>0.2$ (shaded histogram) and sources with $\mathrm{L}_{\mathrm{X}}<10^{43} \mathrm{erg} \mathrm{s}^{-1}$ and difference in frac $\mathrm{AGN}<-0.2$. Thus, we conclude that the addition of the X-ray flux in the SED fitting results in more robust AGN fraction measurements while its effect on frac $_{\mathrm{AGN}}$ is small and depends on the AGN power.

\subsection{The effect of $X$-ray flux on the estimation of the $\mathrm{L}_{2500 \AA}$ and AGN type}

Another important parameter of the SED fitting is the intrinsic $\mathrm{L}_{2500 \AA}$ luminosity. Additionally to the reasons mentioned in section 3.2. $L_{2500 \AA}$ is also a proxy of the AGN power. Here, we study the effect of the X-ray flux on the estimation of $L_{2500 \AA}$. Fig. 10 (left panel) compares the $\mathrm{L}_{2500 \AA}$ estimations with and without X-ray flux in the SED, for type 1 and 2 sources. The classification is based on their inclination angle values, as calculated by X-CIGALE. 2264 sources satisfy our selection criteria, described in section 3.3 , in both runs (with and without Xray flux). In Fig. 10 we have considered only the 1941 sources 

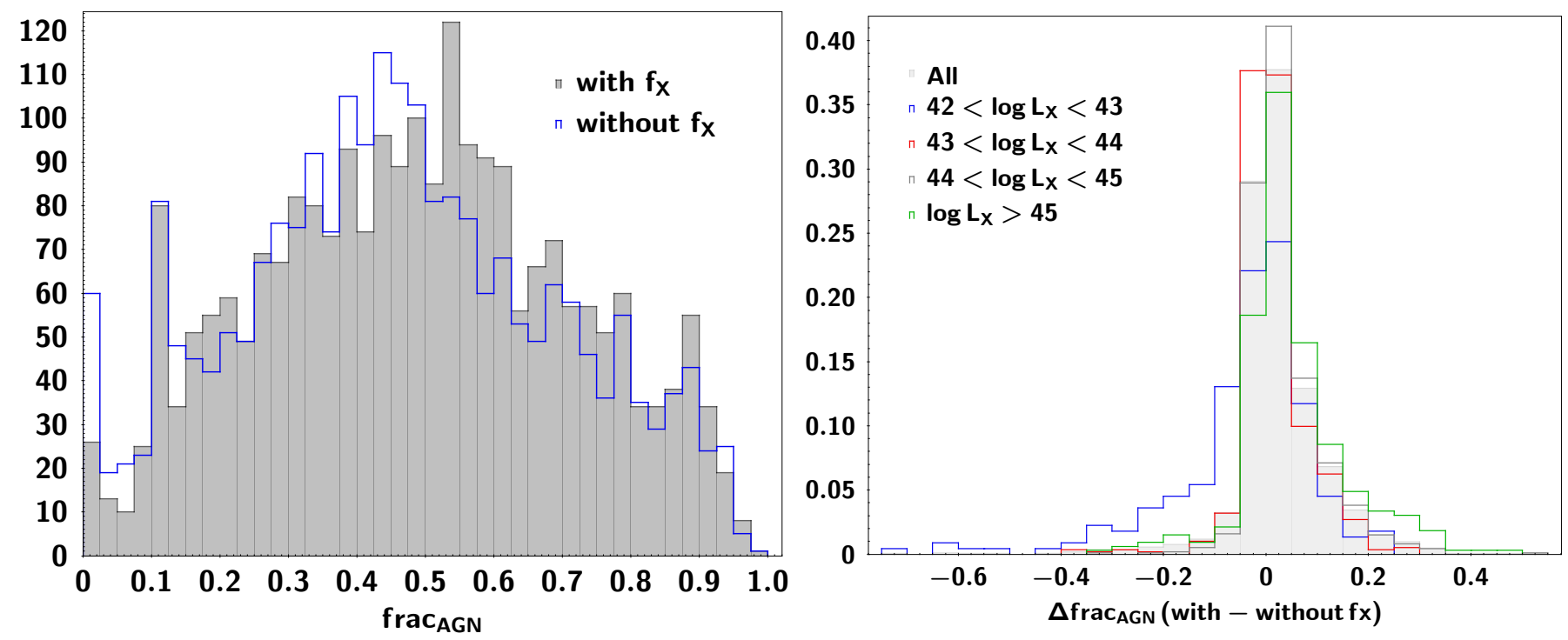

Fig. 8: Left: distribution of the AGN fraction, with X-ray flux (black shaded area) and without X-ray flux (blue histogram). Right: distribution of the difference of the AGN fraction, with and without X-ray flux, for different luminosity bins. All distributions are normalised to unity. A tail appears at positive values, i.e., the AGN fractions are higher when X-ray flux is included in the SED fitting, for AGN with $\mathrm{L}_{X}>10^{43} \mathrm{erg} \mathrm{s}^{-1}$ that becomes more prominent for the most luminous sources $\left(\mathrm{L}_{\mathrm{X}}>10^{45} \mathrm{erg} \mathrm{s}^{-1}\right)$. On the other hand, there is a tail at negative values, i.e. the AGN fractions are lower with X-ray flux, for the fainter $\mathrm{AGN}\left(\mathrm{L}_{\mathrm{X}}<10^{43} \mathrm{erg} \mathrm{s}^{-1}\right.$, blue line).

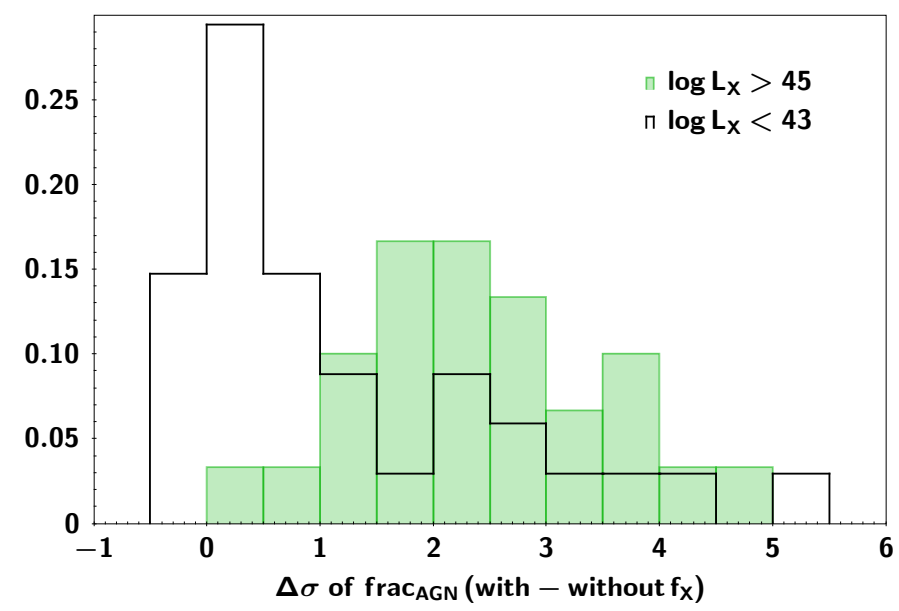

Fig. 9: The difference of the significance, $\Delta \sigma$, of the AGN fraction estimations with and without $\mathrm{X}$-ray information, for sources with $\mathrm{L}_{\mathrm{X}}>10^{45} \mathrm{erg} \mathrm{s}^{-1}$ and difference in frac $\mathrm{AGN}>0.2$ (shaded histogram) and sources with $\mathrm{L}_{\mathrm{X}}<10^{43} \mathrm{erg} \mathrm{s}^{-1}$ and difference in frac $_{\mathrm{AGN}}<-0.2$. The statistical significance of the AGN fraction measurements improves significantly with the inclusion of the X-ray flux, especially for the most luminous AGN.

(86\%) for which the inclination angle from the best fit has the same value in the two runs. For both types, we observe a scatter, but no systematic effect between the two estimations.

$1810 / 2264$ AGN are classified as type 1 with the X-ray flux, but $47(\sim 2.5 \%)$ become type 2 without the X-ray flux. On the other hand, from the 454/2264 sources classified as type 2 with $f_{X}, 276(\sim 60 \%)$ change to type 1 without X-ray flux. The 323 $(47+276)$ sources with different classification in the two runs, are shown in the right panel of Fig. 10 X-CIGALE increases $\mathrm{L}_{2500 \AA}$ when the type changes from type 1 to type 2 with the inclusion of the X-ray flux and lowers $\mathrm{L}_{2500} \AA$ when the type changes from type 2 to type 1 with $f_{X}$. This result is expected, considering the way that X-CIGALE works. (mid-) IR emission is considered anisotropic, i.e., it depends on the viewing angle. The same source will have lower IR flux when viewed edge on (type 2) compared to face on (see Fig. 4 in Stalevski et al. 2012). On the other hand, X-ray flux is considered isotropic. Therefore, a type 2 source will have a higher $\frac{L_{X} \text {,intrinsic }}{L_{I R}}$ (and $\frac{L_{2500 \AA, \text { intrinsic }}}{L_{I R}}$ ) than a type 1 source. For a given observe IR emission, X-CIGALE will decrease/increase the intrinsic accretion power (and thus the intrinsic $L_{2500 \AA}$ ) depending on whether the source is type $1 / 2$.

Our analysis revealed that the inclusion of the X-ray information does not significantly affect the classification of sources that are type 1 based on the run with $f_{X}$, but it does change the characterisation of the majority of the AGN classified as type 2 with X-ray flux. To investigate further, the different classification for the 276 AGN classified as type 2 when the X-ray information is included in the SED, but classified as type 1 without $\mathrm{f}_{X}$, we compare their X-CIGALE classification with that from optical (SDSS) spectra (Menzel et al. 2016). Among those sources that lie at $\mathrm{z}<1, \sim 85 \%$ are classified as Narrow Line AGN (NLAGN2; see section 3.3.2 in Menzel et al.2016), i.e. the optical spectrum agrees with the classification of X-CIGALE when using the X-ray flux. On the other hand, at $\mathrm{z}>1.5, \sim 85 \%$ of the AGN present broad lines in their optical spectra (BLAGN1), i.e. their classification does not agree with that of X-CIGALE when the $\mathrm{X}$-ray flux is included. We conclude that, at $\mathrm{z}<1$, the addition of the X-ray information increases the reliability of the SED fitting regarding the classification type of a source. At high redshifts, though, X-CIGALE seems to misclassify some sources: among the 703 AGN at $\mathrm{z}>1.5$ in our total sample, $103(\approx 15 \%)$ are classified as type 2 with X-ray flux and as type 1 without.

We further investigate plausible reasons for the misclassification of sources at high redshift, when $f_{X}$ is added in the fitting process. The vast majority of these sources lack photometry 

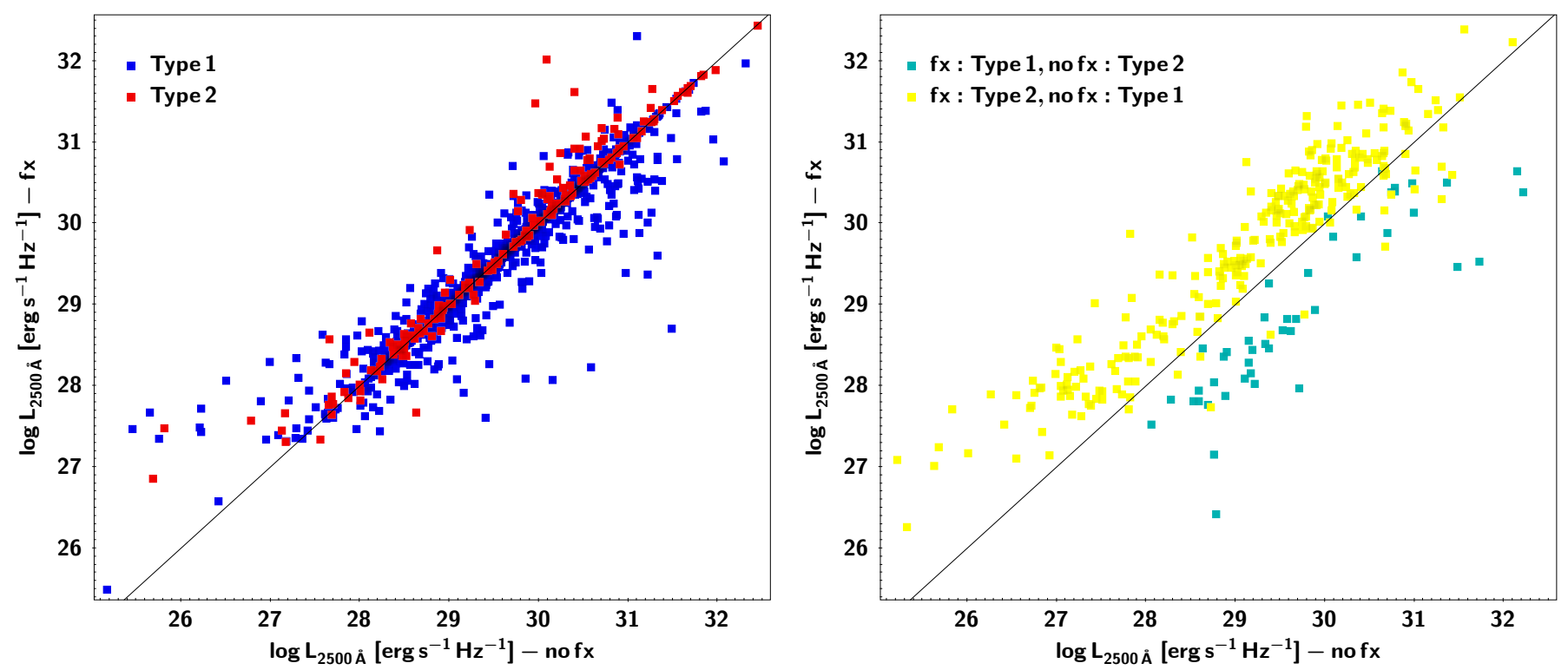

Fig. 10: Comparison of the $\mathrm{L}_{2500} \AA$ values with and without X-ray flux in the fitting process. Left panel: Comparison for sources that have classified with the same type, with and without X-ray flux in the SED fitting process. Blue symbols present the results for type

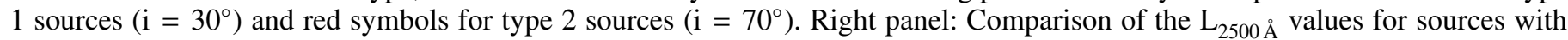
different types from the two runs (with and without X-ray flux). X-CIGALE will decrease/increase the intrinsic accretion power (and thus the intrinsic $L_{2500 \AA}$ ) depending on whether the source is type $1 / 2$.

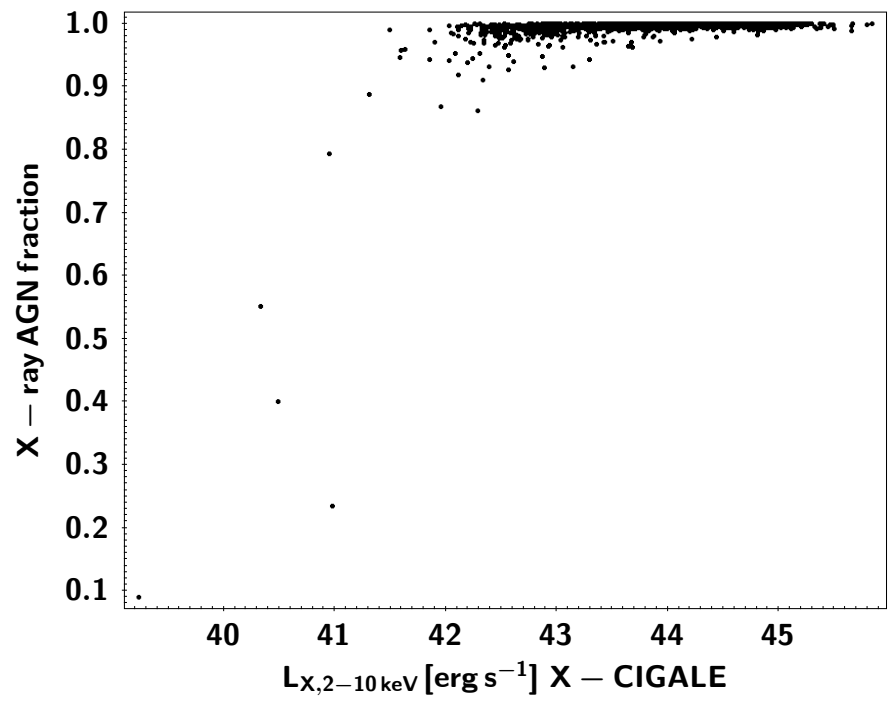

Fig. 11: X-ray AGN fraction vs. X-ray luminosity, estimated by X-CIGALE. The X-ray AGN fraction is defined as the ratio of the X-ray AGN emission to the total X-ray emission of the galaxy (AGN + binaries + hotgas). As expected, for the vast majority of sources the AGN X-ray emission contributes more than $90 \%$ of the total X-ray emission of the galaxy.

above 5 microns. In the absence of IR photometry and without considering the X-ray flux, the introduction of a type 1 component gives a flexibility to fit the UV/optical data while a type 2 component does not contribute to this emission. Thus, without $\mathrm{f}_{\mathrm{X}}, \mathrm{X}$-CIGALE is more likely to classify high redshift sources as type 1 , with the risk to over-fit the UV/optical data. Inclusion of X-ray flux, provides an additional constraint for a type 1 template (via the Just et al. relation), whereas the code can freely

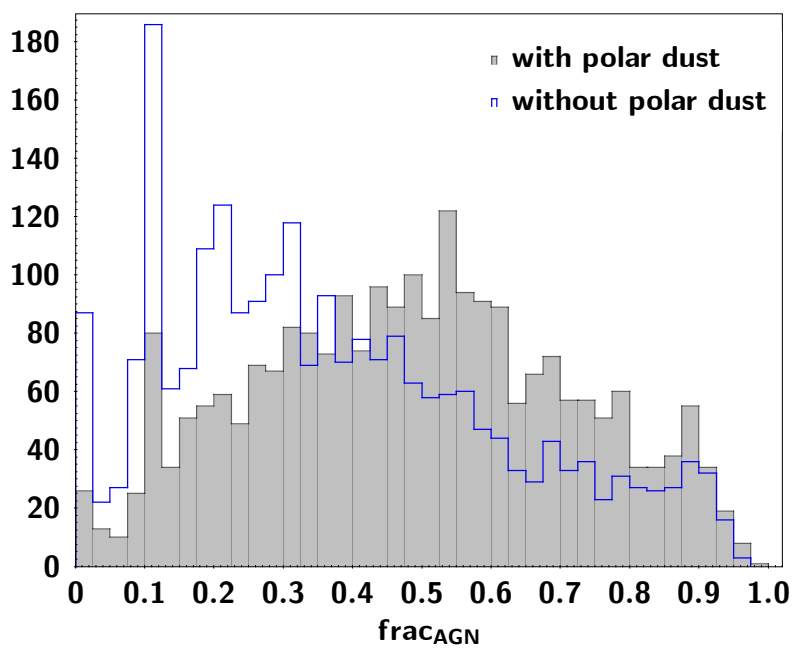

Fig. 12: Distribution of the AGN fraction, with polar dust (black shaded area) and without polar dust (blue histogram). The addition of polar dust in the fitting process increases the AGN fraction.

scale a type 2 template to match any given X-ray flux. Thus, with $f_{X}$, the code will preferentially classify sources as type 2 . In this configuration (SED with only UV/optical data), the difference between a type 1 and a type 2 component is not meaningful.

We conclude, that the misclassification of AGN at high redshifts is mostly due to lack of available IR photometry which makes the type assignation almost unconstrained. Future surveys (JWST; Gardner et al. 2006) could provide mid-IR photometry for these distant AGN. 


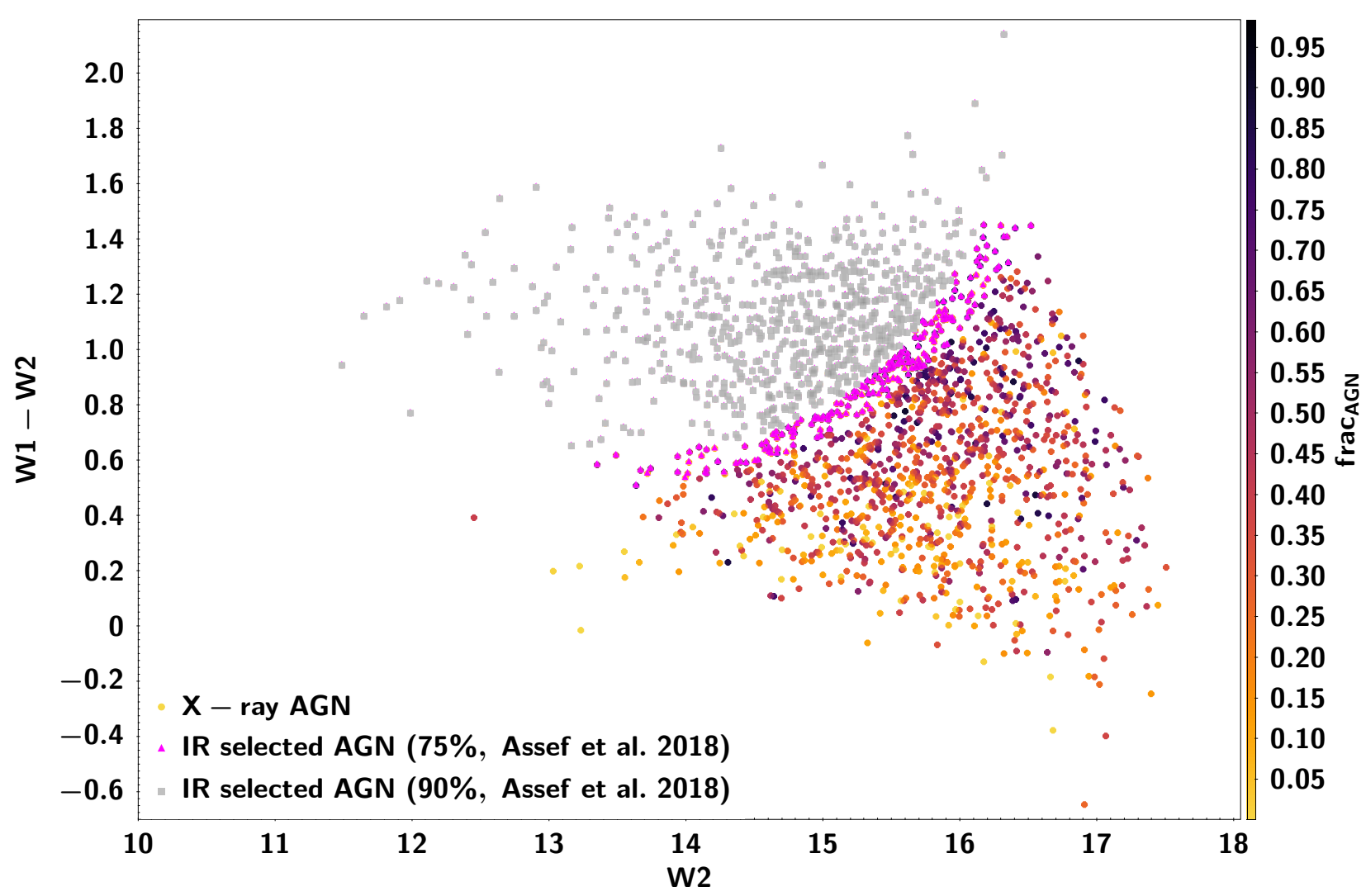

Fig. 13: W1-W2 vs. W2 for the X-ray AGN in our sample (circles). Sources selected as IR AGN with $75 \%$ and $90 \%$ confidence, using the criteria of Assef et al. 2018 are in magenta and grey, respectively. The remaining sources of our X-ray AGN sample are presented with circles, colour coded based on the AGN fraction measurements of X-CIGALE. 97\% of IR selected AGN have frac $_{\mathrm{AGN}}>0.2\left(\sim 92 \%\right.$ with frac $\left.\mathrm{AGN}_{\mathrm{A}}>0.3\right)$. This percentage drops to $71 \%(55 \%$ with frac $\mathrm{AGN}>0.3)$, without polar dust.

Table 5

\begin{tabular}{lcccc}
\hline SED configuration & X-ray AGN & \multicolumn{2}{c}{ IR detected AGN } & non-IR detected AGN \\
& & 75\% Assef & $90 \%$ Assef & \\
& 1956 & $889 / 1956(45 \%)$ & $689 / 1956(35 \%)$ & $1067 / 1956(55 \%)$ \\
& & & & \\
Polar dust $\left(\mathrm{frac}_{\mathrm{AGN}}>0.2\right)$ & & $851 / 889(96 \%)$ & $671 / 689(97 \%)$ & $906 / 1067(85 \%)$ \\
Polar dust $\left(\mathrm{frac}_{\mathrm{AGN}}>0.3\right)$ & $795 / 889(90 \%)$ & $631 / 689(92 \%)$ & $789 / 1067(74 \%)$ \\
& & & \\
w/out polar dust $\left(\mathrm{frac}_{\mathrm{AGN}}>0.2\right)$ & & $740 / 889(83 \%)$ & $591 / 689(86 \%)$ & $757 / 1067(71 \%)$ \\
w/out polar dust $\left(\mathrm{frac}_{\mathrm{AGN}}>0.3\right)$ & $603 / 889(68 \%)$ & $484 / 689(70 \%)$ & $586 / 1067(55 \%)$ \\
\hline
\end{tabular}

\subsection{Ability to quantify the AGN contribution to the total galaxy $X$-ray emission}

Star-forming galaxies may emit X-rays that originate from X-ray binaries, supernovae remnants and hot gas (e.g. Fabbiano 1989). Previous studies have shown that X-ray luminosity can be used as a proxy of SFR (e.g. Ranalli et al. 2003, Laird et al. 2005). However, in X-ray luminous AGN, like those used in this study, the X-ray emission predominately originates from the supermassive black hole and therefore these systems are usually identified and excluded by studies that use the X-ray emission to trace star formation (e.g. Persic \& Rephaeli 2002, Laird et al. 2005).

As already mentioned, X-CIGALE can also model the X-ray emission that originates from low- and high-mass X-ray binaries and hot gas. Thus, in a similar manner to the frac $\mathrm{AGN}_{\mathrm{AG}}$ that quantifies the contribution of the AGN IR emission to the total galaxy IR emission, we define the X-ray AGN fraction as the ratio of the X-ray AGN emission to the total X-ray emission of the galaxy (AGN + binaries + hotgas). In Fig. 11, we plot the X-ray AGN fraction as a function of the X-ray luminosity, estimated by X-CIGALE. As expected, for the vast majority of sources the AGN X-ray emission contributes more than $90 \%$ of the total Xray emission of the galaxy.

Therefore, X-CIGALE by modelling the X-ray emission and its various components offers the capability of estimating the SFR of galaxies not only from IR indicators but also from Xrays, in those systems that are not AGN dominated.

\subsection{Polar dust and AGN fraction}

Another important feature of X-CIGALE is polar dust, quantified with $\mathrm{E}_{\mathrm{B}-\mathrm{V}}$, as a free parameter to account for dust extinction 


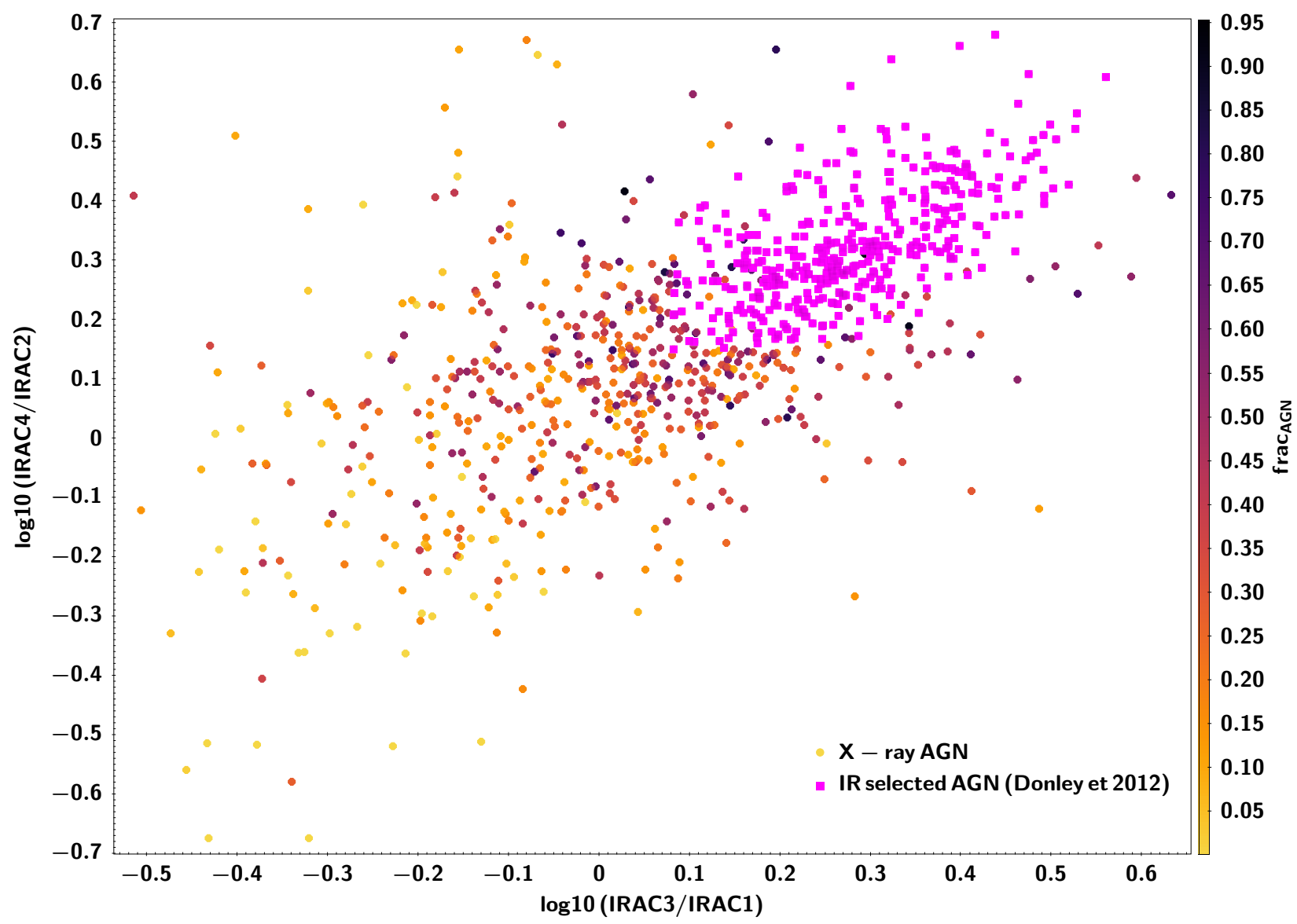

Fig. 14: Colour-colour distribution of the X-ray AGN in our sample (circles), using Spitzer photometry. Circles are colour coded based on the AGN fraction estimations of X-CIGALE. Sources selected as IR AGN using the colour criteria of Donley et al. 2012, are marked with purple squares.

in all AGN, regardless of their classification into type 1 and 2 (see section 3.2.2). In section A.3, we test whether X-CIGALE can successfully constrain $E_{B-V}$. Here, we study the effect of adding polar dust as a free parameter to the SED fitting results, on the AGN fraction measurements.

Fig. 12, presents the distribution of the AGN fraction with polar dust (black shaded area) and without polar dust (blue histogram). In both runs, X-ray flux is included. The addition of polar dust in the fitting process increases the AGN fraction. The mean frac $_{\mathrm{AGN}}$ is $0.37 \pm 0.12$ without polar dust and $0.49 \pm 0.14$ with the addition of polar dust. A similar increase is observed when the two runs do not include $f_{X}$. This trend is also similar regardless of the AGN type, classified based on the inclination angle. The increase of the AGN fraction is expected since the introduction of polar dust allows X-CIGALE to account for obscuring material in the poles of AGN.

\subsection{Polar dust and AGN type}

Repeating the same exercise as for the inclusion of the X-ray flux, we examine whether there are sources for which the classification, based on the estimated inclination angle, changes with the introduction of polar dust ( $f_{X}$ is not included in these runs). Our analysis shows that only $0.01 \%$ of the AGN classified as type 2 with polar dust, change classification without it. However, $14 \%$ of the sources characterised as type 1 when polar dust is included, change to type 2 without polar dust. Addition of at- tenuation by polar dust, provides X-CIGALE the flexibility to attribute part of the absorption to polar dust.

We compare the classification of those sources that their type changes from type 1 to type 2 when we ignore polar dust, with the classification from optical SDSS spectra. 70\% of the AGN present broad lines in the optical continuum. We conclude that the introduction of polar dust as a free parameter in the SED fitting process, improves the accuracy of X-CIGALE in the classification type of the sources.

\subsection{Polar dust and AGN detection efficiency}

$\mathrm{X}$-ray detection provides the most reliable method to identify AGN. Although, X-rays have proven efficient in penetrating the intervening absorbing material (e.g. Luo et al. 2008), even hard $\mathrm{X}$-rays are absorbed by huge amounts of gas and dust. Thus, Xray selected AGN are biased against the most heavily absorbed sources. On the other hand, mid-IR surveys are less affected by extinction. The material that absorbs AGN radiation even at hard $\mathrm{X}$-ray energies, is heated by the AGN and re-emits at infrared wavelengths. Therefore, mid-IR selected AGN can detect AGN that are missed by X-ray surveys (e.g. Georgantopoulos et al. 2008, Fiore et al. 2009). Spitzer, was the first infrared survey that was used to select AGN via colour selection criteria (e.g. Stern et al. 2005; Donley et al. 2012). Later. these techniques were adapted and used for WISE. For instance, Mateos et al. (2012) used a AGN selection method, based on three WISE colours. Assef et al. (2018), used the W1 and W2 criteria of Stern et al. 
(2012) and extended them to fainter magnitudes. However, infrared selection techniques are biased against low luminosity AGN. In a recent study, Pouliasis et al. (2020) showed that SED decomposition can provide a complementary tool to the X-ray and IR selection techniques, since it makes use of large wavelength ranges to efficiently disentangle accretion from star formation (Ciesla et al. 2015, Dietrich et al. 2018, Małek et al. 2018).

Our AGN sample consists of X-ray selected sources with high X-ray luminosity $\left(\mathrm{L}_{\mathrm{X}}>10^{42} \mathrm{erg} \mathrm{s}^{-1}\right)$, therefore the contamination from non AGN systems should be minimal. Thus, we wish to examine whether the addition of polar dust in the fitting process affects the efficiency of the SED decomposition to find a strong AGN component ( frac $_{\mathrm{AGN}}$ ) and compare it with the efficiency of IR colour selection criteria to detect AGN candidates among X-ray sources.

In Fig. 13, we plot the W1-W2 vs. W2 for the X-ray AGN in our sample. With magenta and grey, we mark the sources that are selected as IR AGN candidates with a confidence level of $75 \%$ and $90 \%$, respectively, using the Assef et al. (2018) criteria. Circles present all the X-ray AGN in our dataset, detected by WISE, colour-coded based on the AGN fraction measurements of X-CIGALE. For estimating them, we have included the polar dust as a free parameter but their X-ray flux has been ignored. There are 1956 sources with WISE counterparts that also fulfill our selection criteria (section 3.3). 689/1956 ( 35\%) are detected by Assef et al. with $90 \%$ confidence $(889 / 1956 \approx 45 \%$, with $75 \%$ confidence). 671 out of the 689 sources ( $\sim 97 \%)$, have $\operatorname{frac}_{\mathrm{AGN}}>0.2(631 / 689 \approx 92 \%$ with frac $\mathrm{AGN}>0.3)$, i.e. the SED decomposition reveals strong AGN contribution in the IR emission of the system. In section A.2 we examine how many of the sources with measured frac AGN $>0.2$ (and frac ${ }_{\mathrm{AGN}}>0.3$ ) have true frac $_{\mathrm{AGN}}<0.2$, i.e. the AGN fraction has been miscalculated and the AGN contribution in the IR emission of the galaxy is weaker than assumed. Our analysis shows $<10 \%$ contamination from such systems (Fig. A.3). Among IR selected AGN with $75 \%$ confidence, the numbers are $851 / 889 \approx 96 \%$ with frac $_{\mathrm{AGN}}>0.2\left(795 / 889 \approx 90 \%\right.$ with $\left.\operatorname{frac}_{\mathrm{AGN}}>0.3\right)$. Therefore, X-CIGALE finds a strong AGN component in more than $90 \%$ of the IR selected AGN. However, as we notice in Fig. 13 there is a large number of fainter AGN that are missed by IR selection colour criteria, but the SED fitting finds strong AGN IR emission. Specifically, $85 \%$ of the sources have $\operatorname{frac}_{\mathrm{AGN}}>0.2$ and $74 \%$ have frac $\operatorname{AGN}_{\mathrm{A}}>0.3$. Thus, even considering the more strict criterion of frac $_{\mathrm{AGN}}>0.3$, SED decomposition manages to detect a larger fraction of X-ray selected AGN than IR colour criteria $(74 \%$ vs. $45 \%)$ while at the same time it also uncovers $>90 \%$ of the IR colour selected AGN. Repeating the same exercise without the inclusion of polar dust in the SED fitting process yields, $71 \%$ sources with frac AGN $>0.2$ and $55 \%$ with frac $_{\mathrm{AGN}}>0.3$, among the non-IR detected AGN. Thus, the addition of polar dust allows SED decomposition to uncover a larger fraction of X-ray AGN. The results are summarised in Table 5 . On the other hand, the addition of the X-ray flux only marginally improves the AGN detection efficiency. Specifically, among the non-IR selected AGN, modelling the SEDs with X-ray flux and polar dust, yields $87 \%$ of the sources with frac $_{\mathrm{AGN}}>0.2$ and $75 \%$ with frac $_{\mathrm{AGN}}>0.3$, compared to $85 \%$ and $74 \%$, respectively, using only the polar dust in the fitting process.

In Fig. 14, we repeat the same exercise using the Spitzer colour selection criteria of Donley et al. (2012). 968 AGN in our sample are detected by Spitzer and follow our selection criteria (section 3.3). 409/968 (42\%) are IR selected AGN, based on the criteria presented by Donley et al. (2012). $98 \%$ of these
IR selected AGN have frac $\operatorname{AGN}>0.2\left(92 \%\right.$ with frac $\left._{\mathrm{AGN}}>0.3\right)$. Among the 968 sources with Spitzer counterparts, $\sim 80 \%$ have frac $_{\mathrm{AGN}}>0.2$ and $\sim 67 \%$ have frac $\mathrm{AGN}>0.3$. These numbers drop to $\sim 65 \%$ and $\sim 49 \%$, when polar dust is not included in the SED fitting process.

From this part of the analysis, we conclude that the addition of polar dust improves the ability of the algorithm to disentangle the AGN and host galaxy IR emission and thus increases the efficiency of the SED decomposition to detect a strong AGN component.

\section{Summary}

In this work, we use the SED fitting code X-CIGALE to model the SEDs of $~ 2500$ X-ray AGN in the XMM-XXL field. XCIGALE has some important features to model the AGN emission. It accounts for polar-dust extinction that is commonly found, especially in the case of X-ray selected AGN and includes $\mathrm{X}$-ray data in the SED fitting process.

Our analysis shows that the estimated $\mathrm{L}_{2500 \AA}$ and $\mathrm{L}_{2 \mathrm{keV}}$ follow the adopted Just et al relation, as well as other similar relations in the literature (Lusso \& Risaliti 2016). The SED fitting results are not sensitive to the choice of the extinction law used and the temperature of polar dust. This result holds for our Xray dataset and the available photometry. The addition of far-IR (Herschel) photometry does not statistically affect the polar dust and AGN fraction measurements, but, it slightly increases the statistical significance of the latter (from $3.6 \sigma$ to $4.5 \sigma$ ).

About half of the sources identified as type 2 with the addition of X-ray flux, are found as type 1 in the absence of Xray information. Visual inspection of randomly selected optical (SDSS) spectra, revealed that for $\sim 85 \%$ of the AGN that lie at $\mathrm{z}<1$, the optical spectrum agrees with the X-CIGALE's classification when using $\mathrm{f}_{X}$. However, at higher redshifts $(z>1.5)$ lack of available IR photometry does not allow the algorithm to classify sources in a robust manner. Inclusion of polar dust also improves the agreement of the AGN type classification between $\mathrm{X}$-CIGALE and optical spectra. We conclude that the addition of the X-ray flux and polar dust in the fitting process, improves the accuracy of the classification of AGN into type 1 and 2.

Compared to IR selection techniques (Donley et al. 2012, Assef et al. 2018), X-CIGALE recovered $>90 \%$ of the IR selected AGN. Among the X-ray detected AGN that are not IR selected, SED decomposition attributes a large AGN component in the IR emission of the system ( frac $_{\mathrm{AGN}}>0.2$ ) for $\sim 85 \%$ of them. This number drops to $\sim 70 \%$, when polar dust is not included in the SED modelling. Thus, addition of polar dust improves the efficiency of the SED decomposition to detect AGN.

One of the most important strengths of SED fitting is that it allows disentangling the IR emission of AGN from that of the host galaxy, quantified by frac ${ }_{A G N}$ in X-CIGALE. The new features of X-CIGALE improve the AGN fraction measurements. Specifically, the addition of the X-ray flux, improves the statistical significance of the AGN fraction measurements, in particular for luminous $\left(\mathrm{L}_{\mathrm{X}}>10^{45} \mathrm{erg} \mathrm{s}^{-1}\right)$ sources (Fig. 9). The addition of polar dust increases the frac $\mathrm{AGN}_{\mathrm{A}}$ estimations, since the AGN contribute more to the IR emission of the system.

The conclusions of our analysis hold under the condition that (mid-) IR photometry is available in the SED fitting process. Lack of IR photometry may result in unreliable X-ray luminosity calculations, lower and less robust AGN fraction estimates and AGN type misclassifications.

Acknowledgements.

The authors thank the anonymous referee for their comments. 
The authors thank Raphael Shirley and Yannick Roehlly for their help to retrieve IR fluxes on the XMM-LSS field.

GM acknowledges support by the Agencia Estatal de Investigación, Unidad de Excelencia María de Maeztu, ref. MDM-2017-0765.

MB acknowledges FONDECYT regular grant 1170618

$\mathrm{XXL}$ is an international project based around an $X M M$ Very Large Programme surveying two $25 \mathrm{deg}^{2}$ extragalactic fields at a depth of $\sim 6 \times 10^{-15} \mathrm{erg}$ $\mathrm{cm}^{-2} \mathrm{~s}^{-1}$ in the [0.5-2] keV band for point-like sources. The XXL website is http://irfu.cea.fr/xxl// Multi-band information and spectroscopic follow-up of the $\mathrm{X}$-ray sources are obtained through a number of survey programmes, summarised at http://xxlmultiwave.pbworks.com/.

This research has made use of data obtained from the 3XMM XMM-Newton serendipitous source catalogue compiled by the 10 institutes of the XMMNewton Survey Science Centre selected by ESA.

This work is based on observations made with XMM-Newton, an ESA science mission with instruments and contributions directly funded by ESA Member States and NASA.

Funding for the Sloan Digital Sky Survey IV has been provided by the Alfred P. Sloan Foundation, the U.S. Department of Energy Office of Science, and the Participating Institutions. SDSS-IV acknowledges support and resources from the Center for High-Performance Computing at the University of Utah. The SDSS web site is www.sdss.org

SDSS-IV is managed by the Astrophysical Research Consortium for the Participating Institutions of the SDSS Collaboration including the Brazilian Participation Group, the Carnegie Institution for Science, Carnegie Mellon University, the Chilean Participation Group, the French Participation Group, HarvardSmithsonian Center for Astrophysics, Instituto de Astrofísica de Canarias, The Johns Hopkins University, Kavli Institute for the Physics and Mathematics of the Universe (IPMU) / University of Tokyo, Lawrence Berkeley National Laboratory, Leibniz Institut für Astrophysik Potsdam (AIP), Max-Planck-Institut für Astronomie (MPIA Heidelberg), Max-Planck-Institut für Astrophysik (MPA Garching), Max-Planck-Institut für Extraterrestrische Physik (MPE), National Astronomical Observatories of China, New Mexico State University, New York University, University of Notre Dame, Observatário Nacional / MCTI, The Ohio State University, Pennsylvania State University, Shanghai Astronomical Observatory, United Kingdom Participation Group, Universidad Nacional Autónoma de México, University of Arizona, University of Colorado Boulder, University of Oxford, University of Portsmouth, University of Utah, University of Virginia, University of Washington, University of Wisconsin, Vanderbilt University, and Yale University.

This publication makes use of data products from the Wide-field Infrared Survey Explorer, which is a joint project of the University of California, Los Angeles, and the Jet Propulsion Laboratory/California Institute of Technology, funded by the National Aeronautics and Space Administration.

The VISTA Data Flow System pipeline processing and science archive are described in Irwin et al. (2004), Hambly et al. (2008) and Cross et al. (2012). Based on observations obtained as part of the VISTA Hemisphere Survey, ESO Program, 179.A-2010 (PI: McMahon). We have used data from the 3rd data release. This work is based [in part] on observations made with the Spitzer Space Telescope, which was operated by the Jet Propulsion Laboratory, California Institute of Technology under a contract with NASA

The project has received funding from Excellence Initiative of Aix-Marseille University - AMIDEX, a French 'Investissements d'Avenir' programme.

\section{References}

Asmus, D. 2019, Monthly Notices of the Royal Astronomical Society, 489, 2177 Asmus, D., Hönig, S. F., Gandhi, P., Smette, A., \& Duschl, W. J. 2014, Monthly Notices of the Royal Astronomical Society, 439, 1648

Assef, R. J., Stern, D., Noirot, G., et al. 2018, The Astrophysical Journal Supplement Series, 234, 23

Bongiorno, A., Merloni, A., Brusa, M., et al. 2012, Monthly Notices of the Royal Astronomical Society, 427, 3103

Boquien, M., Burgarella, D., Roehlly, Y., et al. 2019, Astronomy \& Astrophysics, 622, A103

Brandt, W. N. \& Alexander, D. M. 2015, A\&A Rev., 23, 1

Bruzual, G. \& Charlot, S. 2003, MNRAS, 344, 1000

Burgarella, D., Buat, V., \& Iglesias-Páramo, J. 2005, Monthly Notices of the Royal Astronomical Society, 360, 1413

Calzetti, D., Armus, L., Bohlin, R. C., et al. 2000, ApJ, 533, 682

Caputi, K. I., Cirasuolo, M., Dunlop, J. S., et al. 2011, Monthly Notices of the Royal Astronomical Society, 413, 162

Carnall, A. C., McLure, R. J., Dunlop, J. S., \& Davé, R. 2018, Monthly Notices of the Royal Astronomical Society, 480, 4379

Chabrier, G. 2003, PASP, 115, 763

Ciesla, L. et al. 2015, A\&A, 576, 19

Civano, F., Elvis, M., Brusa, M., et al. 2012, ApJS, 201, 30
Cross, N. J. G. et al. 2012, A\&A, 548, 21

da Cunha, E., Charlot, S., \& Elbaz, D. 2008, Monthly Notices of the Royal Astronomical Society, 388, 1595

Dale, D. A., Helou, G., Magdis, G. E., et al. 2014, ApJ, 784, 83

Dawson, K. S. et al. 2013, AJ, 145, 10

Dietrich, J., Weiner, A. S., Ashby, M. L. N., et al. 2018, Monthly Notices of the Royal Astronomical Society, 480, 3562

Donley, J. L., Koekemoer, A. M., Brusa, M., et al. 2012, ApJ, 748, 142

Eisenstein, D. J. et al. 2011, AJ, 142, 72

Emerson, J., McPherson, A., \& Sutherland, W. 2006, Msngr, 126, 41

Fabbiano, G. 1989, Annual Review of Astronomy and Astrophysics, 27, 87

Feltre, A., Hatziminaoglou, E., Fritz, J., \& Franceschini, A. 2012, MNRAS, 426, 120

Ferrarese, L. \& Merritt, D. 2000, ApJ, 539, 9

Fiore, F., Puccetti, S., Brusa, M., et al. 2009, ApJ, 693, 447

Gardner, J. P., Mather, J. C., Clampin, M., et al. 2006, Space Science Reviews, 123,485

Gaskell, C. M., Goosmann, R. W., Antonucci, R. R. J., \& Whysong, D. H. 2004, The Astrophysical Journal, 616, 147

Georgakakis, A. et al. 2011, MNRAS, 418, 2590

Georgakakis, A. et al. 2017, MNRAS, 469, 3232

Georgantopoulos, I., Georgakakis, A., Rowan-Robinson, M., \& Rovilos, E. 2008, A\&A, 484, 671

Hambly, N. C. et al. 2008, MNRAS, 384, 637

Hatziminaoglou, E., Fritz, J., \& Jarrett, T. H. 2009, Monthly Notices of the Royal Astronomical Society, 399, 1206

Hurley, P. D., Oliver, S., Betancourt, M., et al. 2017, Monthly Notices of the Royal Astronomical Society, 464, 885

Irwin, M. J. et al. 2004, SPIE, 5493, 411

Jaffarian, G. W. \& Gaskell, C. M. 2020, Monthly Notices of the Royal Astronomical Society, 493, 930

Just, D. W., Brandt, W. N., Shemmer, O., et al. 2007, ApJ, 685, 1004

Kaastra, J. S., Kriss, G. A., Cappi, M., et al. 2014, Science, 345, 64

Laird, E. S., Nandra, K., Adelberger, K. L., Steidel, C. C., \& Reddy, N. A. 2005, Monthly Notices of the Royal Astronomical Society, 359, 47

Leja, J., Johnson, B. D., Conroy, C., van Dokkum, P. G., \& Byler, N. 2017, The Astrophysical Journal, 837, 170

Li, J., Xue, Y., Sun, M., et al. 2019, The Astrophysical Journal, 877, 5

Liu, Z., Merloni, A., Georgakakis, A., et al. 2016, MNRAS, 459, 1602

Lonsdale, C. J., Smith, H. E., Rowan-Robinson, M., et al. 2003, Publications of the Astronomical Society of the Pacific, 115, 897

Luo, B., Bauer, F. E., Brandt, W. N., et al. 2008, The Astrophysical Journal Supplement Series, 179, 19

Lusso, E. \& Risaliti, G. 2016, ApJ, 819, 154

Lusso, E. et al. 2012, MNRAS, 425, 623

Magorrian, J. et al. 1998, AJ, 115, 2285

Małek, K., Buat, V., Roehlly, Y., et al. 2018, Astronomy \& Astrophysics, 620, A50

Malizia, A., Bassani, L., Stephen, J. B., Bazzano, A., \& Ubertini, P. 2020, Astronomy \& Astrophysics, 639, A5

Masoura, V. A., Georgantopoulos, I., Mountrichas, G., et al. 2020, Astronomy \& Astrophysics, 638, A45

Masoura, V. A., Mountrichas, G., Georgantopoulos, I., et al. 2018, A\&A, 618, 31

Mateos, S., Alonso-Herrero, A., Carrera, F. J., et al. 2012, MNRAS, 426, 3271

Menzel, M.-L. et al. 2016, MNRAS, 457, 110

Morganti, R. 2017, Nature Astronomy, 1, 39

Mukai, K. 1993, Legacy, 3, 21

Noll, S. et al. 2009, A\&A, 507, 1793

Oliver, S. J., Bock, J., Altieri, B., et al. 2012, Monthly Notices of the Royal Astronomical Society, 424, 1614

Oliver, S. J. et al. 2012, MNRAS, 424, 1614

Park, T., Kashyap, V. L., Siemiginowska, A., et al. 2006, The Astrophysical Journal, 652, 610

Persic, M. \& Rephaeli, Y. 2002, Astronomy \& Astrophysics, 382, 843

Pierre, M. et al. 2016, A\&A, 592, 1

Pouliasis, E., Mountrichas, G., Georgantopoulos, I., et al. 2020, Monthly Notices of the Royal Astronomical Society, 495, 1853

Prevot, M., Lequeux, J., Maurice, E., Prevot, L., \& Rocca-Volmerange, B. 1984, A\&A, 132, 389

Ranalli, P., Comastri, A., \& Setti, G. 2003, Astronomy \& Astrophysics, 399, 39

Reichert, G. A., Mushotzky, R. F., Holt, S. S., \& Petre, R. 1985, The Astrophysical Journal, 296, 69

Risaliti, G. \& Lusso, E. 2017, Astronomische Nachrichten, 338, 329

Robotham, A. S. G., Bellstedt, S., del P Lagos, C., et al. 2020, Monthly Notices of the Royal Astronomical Society, 495, 905

Smee, S. et al. 2013, AJ, 146, 32

Stalevski, M., Fritz, J., Baes, M., Nakos, T., \& Popović, L. Č. 2012, Monthly Notices of the Royal Astronomical Society, 420, 2756 
Stalevski, M., Ricci, C., Ueda, Y., et al. 2016, Monthly Notices of the Royal Astronomical Society, 458,2288

Stanley, F., Harrison, C. M., Alexander, D. M., et al. 2018, Monthly Notices of the Royal Astronomical Society, 478, 3721

Stern, D., Assef, R. J., Benford, D. J., et al. 2012, ApJ, 753, 30

Stern, D., Eisenhardt, P., Gorjian, V., et al. 2005, ApJ, 631, 163

Sutherland, W. \& Saunders, W. 1992, MNRAS, 259, 413

Tristram, K. R. W., Burtscher, L., Jaffe, W., et al. 2014, Astronomy \& Astrophysics, 563, A82

Werner, M. W., Roellig, T. L., Low, F. J., et al. 2004, ApJS, 154, 1

Wright, E. L., Eisenhardt, P. R. M., Mainzer, A. K., et al. 2010, AJ, 140, 1868

Yan, L. et al. 2013, AJ, 145, 55

Yang, G., Boquien, M., Buat, V., et al. 2020, Monthly Notices of the Royal Astronomical Society, 491, 740

Yang, G., Brandt, W. N., Alexander, D. M., et al. 2019, Monthly Notices of the Royal Astronomical Society, 485, 3721

Yang, G., Brandt, W. N., Luo, B., et al. 2016, The Astrophysical Journal, 831, 145 


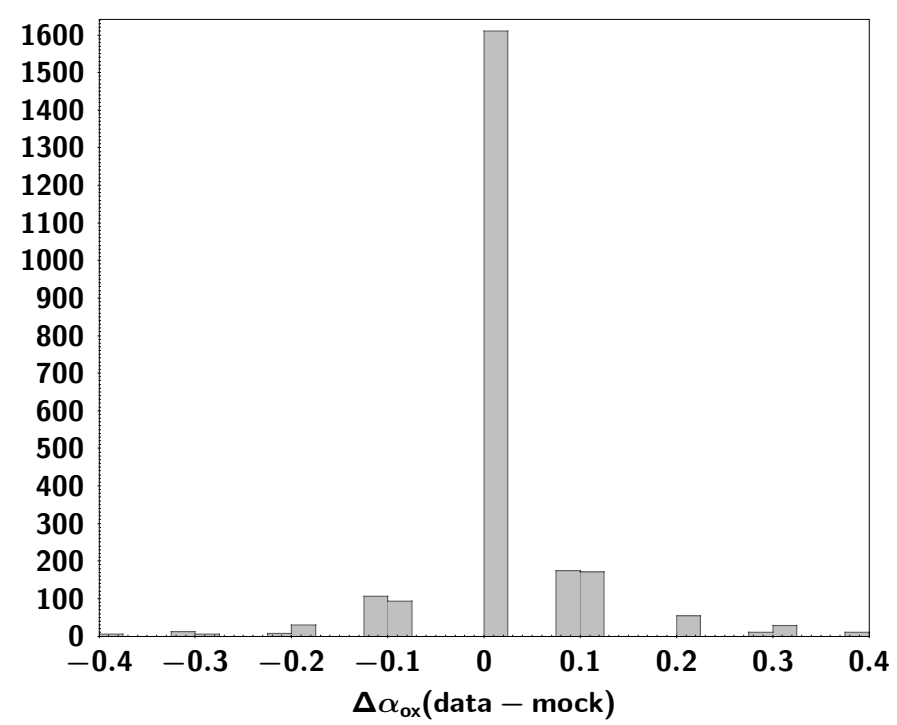

Fig. A.1: The efficiency of X-CIGALE to constrain the $\alpha_{o x}$ parameter, using the mock catalogue (see section 3.2.4). THe distribution highly peaks at zero, with $95 \%$ of the sources to lie within \pm 0.1 . This demonstrates that the algorithm can successfully estimate $\alpha_{o x}$.

\section{Appendix A: Mock catalogue analysis}

As mentioned in section 3.2.4. X-CIGALE offers the option to create and analyse mock catalogues based on the best fit model of each source of the dataset. Here, we use this to assess the efficiency of X-CIGALE in estimating important parameters. Throughout this section, the bayesian estimates of the mock values will be presented.

\section{Appendix A.1: The efficiency of X-CIGALE in estimating $\alpha_{o x}$ and $\mathrm{L}_{2500} \AA$}

In Fig. A.1, we test X-CIGALE's reliability in estimating the $\alpha_{o x}$ parameter, using the results of the mock catalogue. Specifically, we compare the true values of the parameters from the best fit of the dataset to the Bayesian values of the parameter obtained from the fit of the mock catalogue. The distribution of the difference of the two values highly peaks at zero and $\sim 95 \%$ of the sources are within \pm 0.1 . This demonstrates that the algorithm can reliably recover this parameter.

In the same fashion, Fig. A.2, presents the efficiency of XCIGALE to constrain $\mathrm{L}_{2500 \AA}$, with (left panel) and without (right panel) the X-ray flux. When the X-ray flux is included in the $\mathrm{SED}, \mathrm{L}_{2500 \AA}$ is well constrained. When there is no X-ray information in the SED the scatter is larger. This is expected, based on how X-CIGALE calculates this parameter in the absense of the X-ray flux (see section 3.2.2).

\section{Appendix A.2: The efficiency of X-CIGALE in estimating the AGN fraction}

Here, we examine the accuracy of X-CIGALE in the estimation of the AGN fraction. In Fig. A.3, we plot the AGN fraction values estimated by the fit of the mock catalogue vs. the true values, i.e. the values from the best fit of the data. Circles present the mean measurements and their standard deviation is also plotted. Median values are shown by triangles. There is a good agreement between the estimated and the true values.
In Fig. A.4. we plot the difference of the AGN fraction values estimated by fitting the mock catalogue from the true values. We present the results when the X-ray flux has been included in the SED (shaded histograms) and without including the X-ray flux (green lines). The results are split into X-ray luminosity bins. Based on our analysis, X-CIGALE can recover successfully the AGN fractions, regardless of whether the X-ray flux is included in the SED fitting or not, at all X-ray luminosities.

In section 5.6, we examine the fraction of X-ray AGN for which a strong AGN component is measured, i.e. $\operatorname{frac}_{\mathrm{AGN}}>0.2$. In Fig. A.5, we test the level of contamination in these calculations, i.e., the percentage of sources that their true AGN fraction is $<0.2$, albeit the measured frac $_{\mathrm{AGN}}>0.2$. Using a threshold of measured (mock) frac AGN $>0.2$ the contamination is $\approx 10 \%$ which drops to $\sim 6.5 \%$ if we use a higher threshold of frac $_{\mathrm{AGN}}>0.3$. For this calculation we run X-CIGALE with polar dust and without X-ray flux, in accordance with the configuration used in section 5.6 .

\section{Appendix A.3: The efficiency of X-CIGALE in the estimation of polar dust contribution}

To check whether X-CIGALE can successfully constrain the effect of dust extinction by polar dust, in Fig. A.6 we plot the Bayesian estimations of the $\mathrm{E}_{\mathrm{B}-\mathrm{V}}$ parameter for the polar dust vs. its exact value. The scatter ( $1 \sigma$ variations $)$ is substantial. Furthermore, for $\mathrm{E}_{\mathrm{B}-\mathrm{V}}<0.5$ the fit is not sensitive to an incremental increase of the reddening parameter. The sensitivity is better at higher values of $E_{B-V}$, but the parameter is still not determined well. The picture remains the same regardless of whether the Xray flux is taken into account in the SED fitting. These results suggest that it is redundant to use in the SED configuration process, values of $E_{B-V}$ within small intervals and that the estimated values of the parameter are not well constrained. 

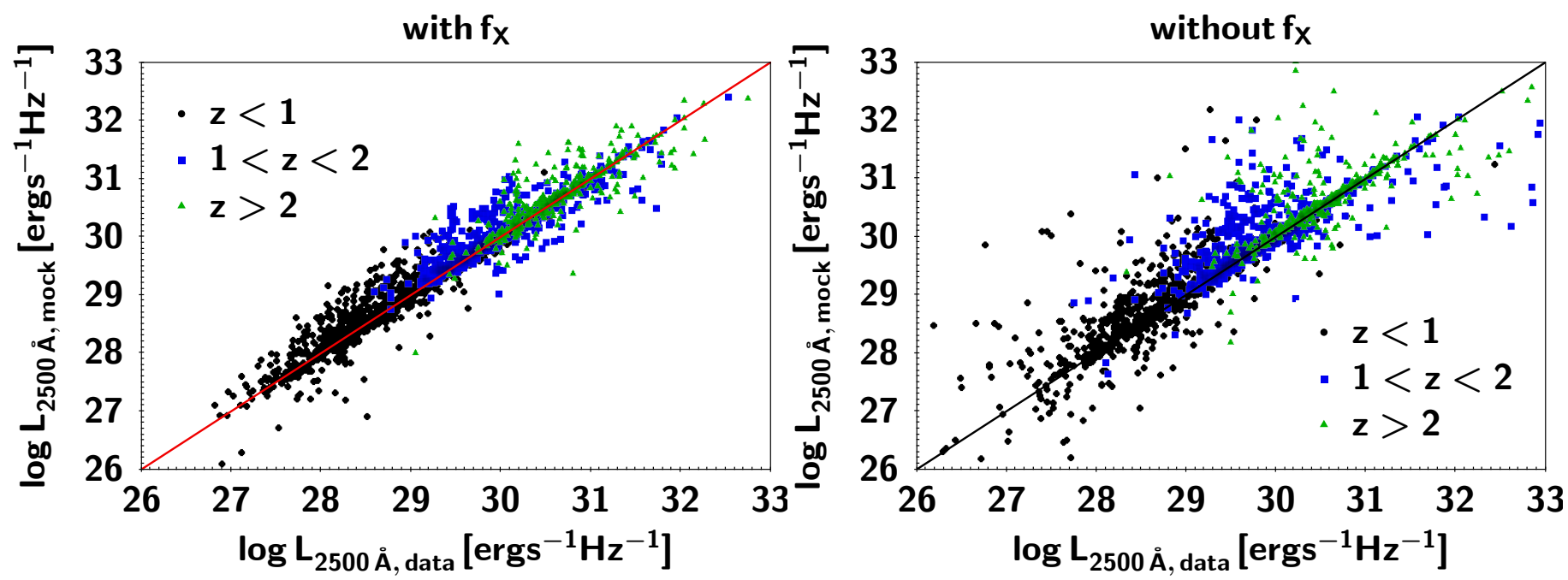

Fig. A.2: Comparison of the $\mathrm{L}_{2500 \AA}$ values estimated by X-CIGALE for the mock sources with the true values (data). Left panel: When the X-ray flux is included in the SED, the $\mathrm{L}_{2500} \AA$ is well constained. Right panel: The X-ray flux is not included in the data. Although, X-CIGALE recovers the parameter successfully, the scatter is larger compared to the results with the X-ray flux.

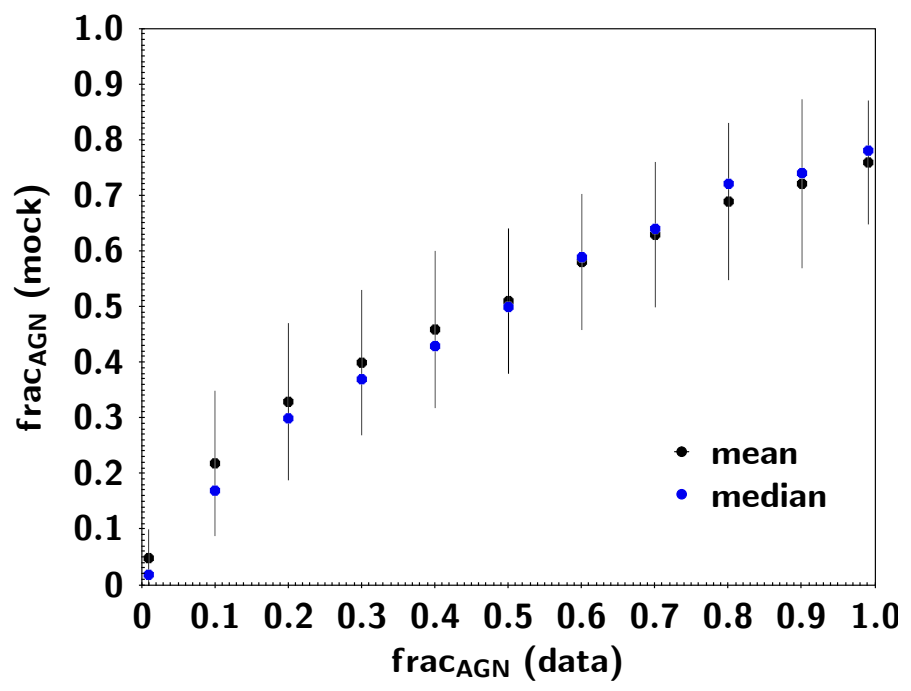

Fig. A.3: AGN fraction values estimated by the fit of the mock catalogue vs. the true values (from the fit of the data). Circles present the mean measurements and their standard deviation is also plotted. Median values are shown by triangles. 

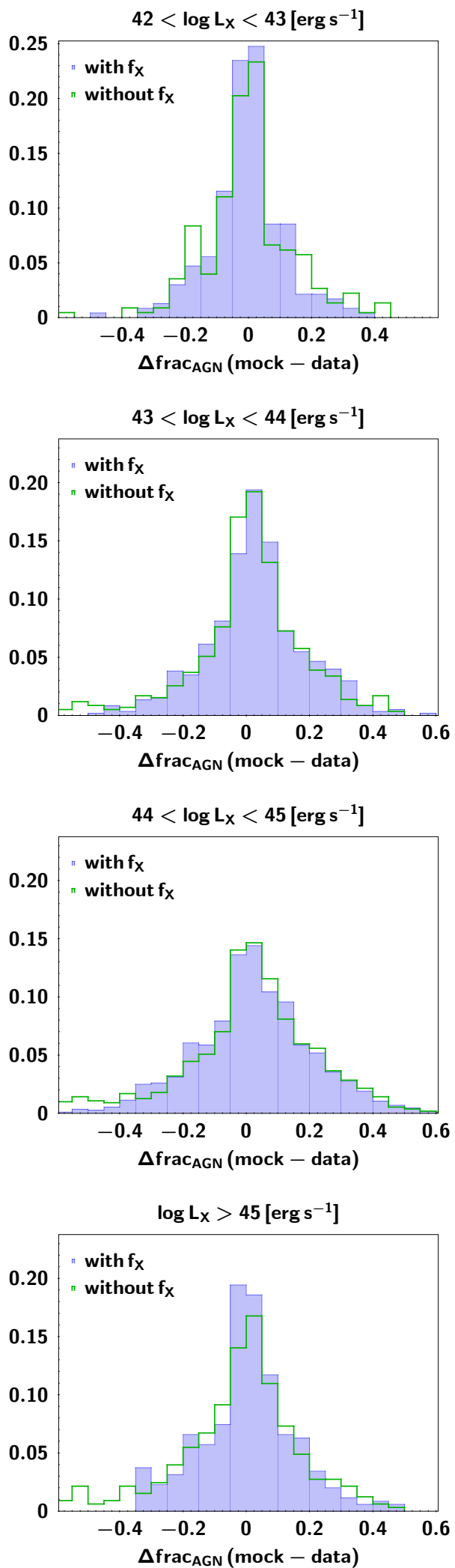

Fig. A.4: The difference of the AGN fraction values estimated by fitting the mock catalogue from the true values. We present the results when the X-ray flux has been included in the SED (shaded histograms) and without including the X-ray flux (green histograms). The results are split into X-ray luminosity bins. XCIGALE can recover successfully the AGN fractions, regardless of whether the X-ray flux is included in the SED fitting or not, at all X-ray luminosities.

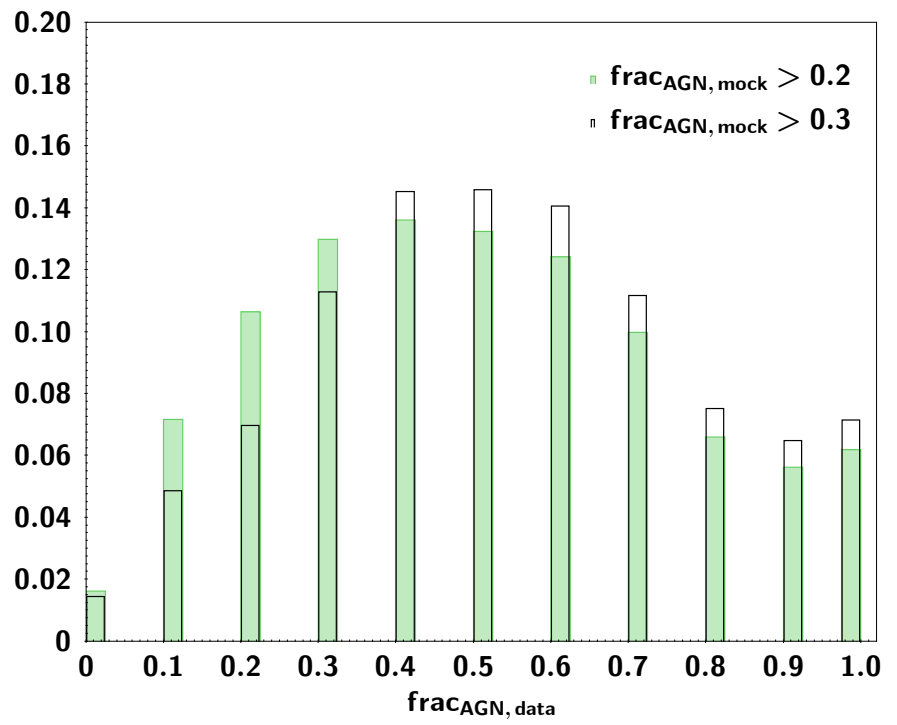

Fig. A.5: Binned measurements of the true AGN fraction values (data), for frac ${ }_{A G N, \text { mock }}>0.2$ (green histogram) and frac $_{\mathrm{AGN} \text {,mock }}>0.3$ (black histogram) from the mock analysis. For sources with frac $_{\mathrm{AGN} \text {,mock }}>0.2$, there is $\approx 10 \%$ contamination, i.e. sources that the AGN component is low frac AGN,data $_{<}$ 0.2 . For sources with frac $_{\mathrm{AGN} \text {,mock }}>0.3$, the contamination is $\sim 6.5 \%$. 
with $f_{X}$

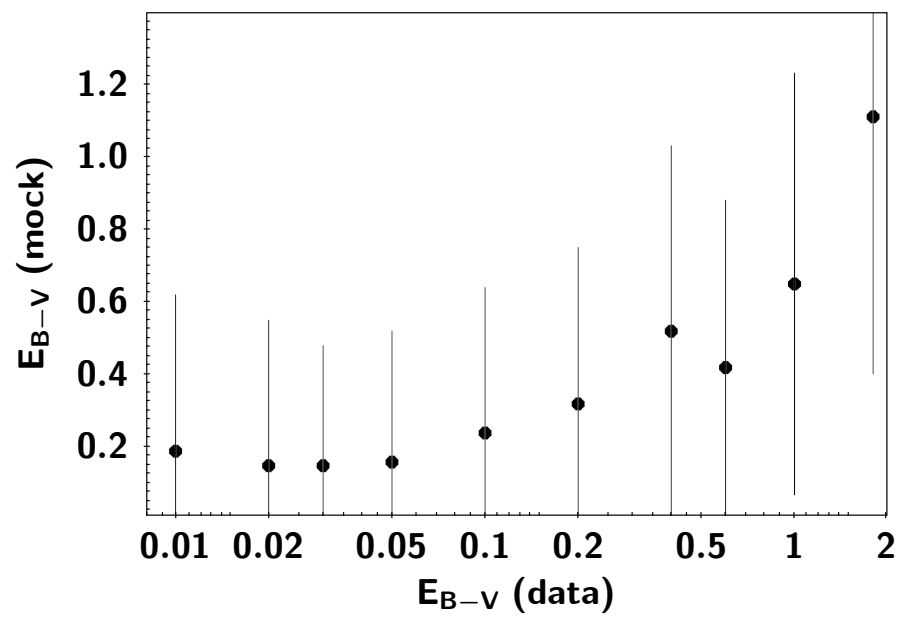

without $f_{X}$

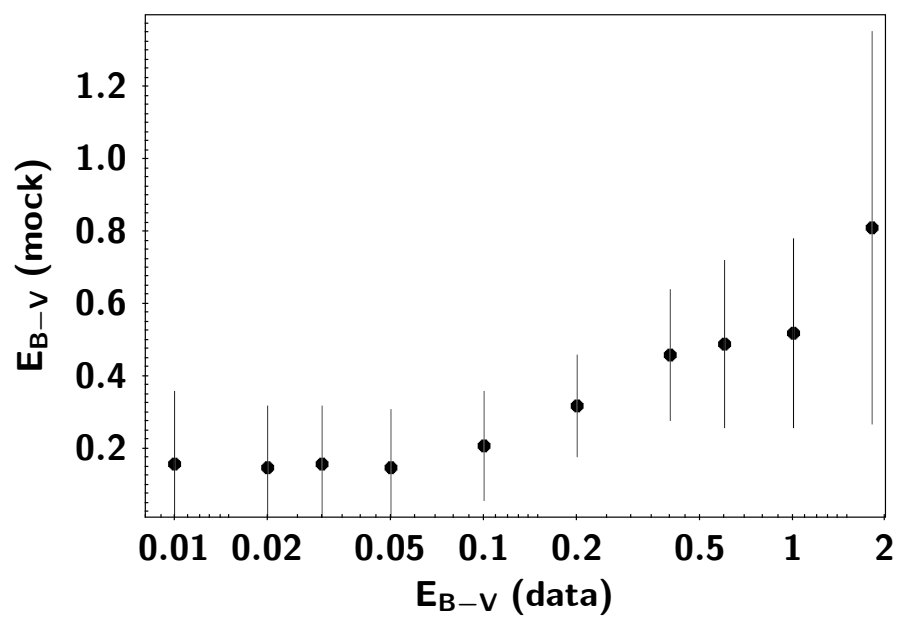

Fig. A.6: Polar dust estimations from the mock sources vs. the input (data) values. Although the $1 \sigma$ deviation is large, mock values increase with the increase of the data values. However, the algorithm is not sensitive to incremental increases of the parameter. The trends are similar regardless, of whether the X-ray flux is included (left panel) or not (right panel) in the fitting process. 